SANDIA REPORT

SAND98-1013 - UC-404

Unlimited Release

Printed May 1998

\section{RECEIVED}

MAY 271998

OSTI

\title{
Cold-Cathodes for Sensors and Vacuum Microelectronics
}

M. P. Siegal, J. P. Sullivan, D. R. Tallant, R. L. Simpson, N. J. DiNardo, T. W. Mercer, L. J. Martinez-Miranda

Prepared by

Sandia National Laboratories

Albuquerque, New Mexico 87185 and Livermore, California 94550

Sandia is a multiprogram laboratory operated by Sandia Corporation, a Lockheed Martin Company, for the United States Department of Energy under Contract DE-AC04-94AL 85000 .

Approved for public release, further dissemination unlimited.

Sandia National Laboratories

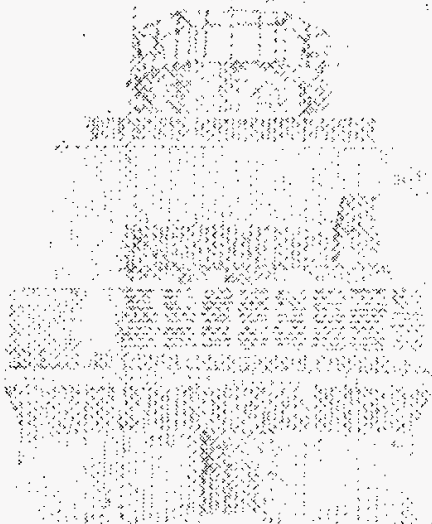

SF29000(8-81)

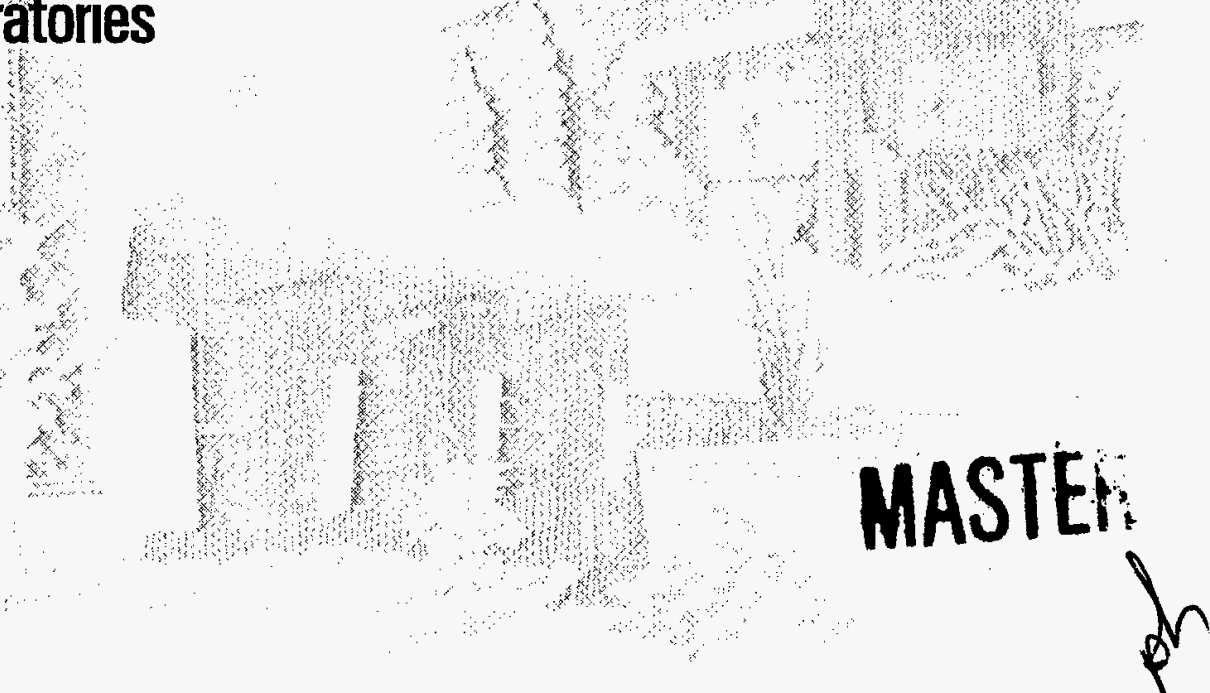

DISTRIBUTIGIS OF THIS EOCUMENT IS UNLAMIED 
Issued by Sandia National Laboratories, operated for the United States Department of Energy by Sandia Corporation.

NOTICE: This report was prepared as an account of work sponsored by an agency of the United States Government. Neither the United States Government nor any agency thereof, nor any of their employees, nor any of their contractors, subcontractors, or their employees, makes any warranty, express or implied, or assumes any legal liability or responsibility for the accuracy, completeness, or usefulness of any information, apparatus, product, or process disclosed, or represents that its use would not infringe privately owned rights. Reference herein to any specific commercial product, process, or service by trade name, trademark, manufacturer, or otherwise, does not necessarily constitute or imply its endorsement, recommendation, or favoring by the United States Government, any agency thereof, or any of their contractors or subcontractors. The views and opinions expressed herein do not necessarily state or reflect those of the United States Government, any agency thereof, or any of their contractors.

Printed in the United States of America. This report has been reproduced directly from the best available copy.

Available to DOE and DOE contractors from

Office of Scientific and Technical Information

P.O. Box 62

Oak Ridge, TN 37831

Prices available from (615) 576-8401, FTS 626-8401

Available to the public from

National Technical Information Service

U.S. Department of Commerce

5285 Port Royal Rd

Springfield, VA 22161

NTIS price codes

Printed copy: A03

Microfiche copy: A01

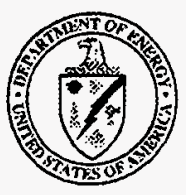




\title{
Cold-Cathodes for Sensors and Vacuum Microelectronics
}

\author{
M. P. Siegal and J. P. Sullivan \\ Advanced Materials and Device Sciences Department \\ D. R. Tallant, R. L. Simpson \\ Surface/Molecular Spectro/Gas Department \\ Sandia National Laboratories, P. O. Box 5800, Albuquerque, NM 87185-1421 \\ N. J. DiNardo and T. W. Mercer \\ Department of Physics and Astronomy \\ Drexel University, Philadelphia, PA \\ L. J. Martinez-Miranda \\ Department of Materials Science and Engineering \\ University of Maryland, College Park, MD
}

\begin{abstract}
The aim of this laboratory-directed research and development project was to study amorphous carbon (a-C) thin films for eventual cold-cathode electron emitter applications. The development of robust, cold-cathode emitters are likely to have significant implications for modern technology and possibly launch a new industry: vacuum micro-electronics (VME). The potential impact of VME on Sandia's National Security missions, such as defense against military threats and economic challenges, is profound. VME enables new microsensors and intrinsically radiation-hard electronics compatible with MOSFET and IMEM technologies. Furthermore, VME is expected to result in a breakthrough technology for the development of high-visibility, low-power flat-panel displays. This work covers four important research areas. First, we studied the nature of the $\mathrm{C}-\mathrm{C}$ bonding structures within these a-C thin films. Second, we determined the changes in the film structures resulting from thermal annealing to simulate the effects of device processing on a-C properties. Third, we performed detailed electrical transport measurements as a function of annealing temperature to correlate changes in transport properties with structural changes and to propose a model for transport in these a-C materials with implications on the nature of electron emission. Finally, we used scanning atom probes to determine important aspects on the nature of emission in a-C.
\end{abstract}




\section{Acknowledgments}

The authors thank T. Friedmann for providing the films used in this study, and N. Missert for performing some of the $\mathrm{x}$-ray reflectivity measurements. Both are former members of the Advanced Materials and Device Sciences Department. We also thank E. Stechel (same department) and P. Schultz of the Computational Materials Science Department for many helpful discussions and their collaborative work studying theoretical structural models for amorphous carbon materials.

We thank R. Dunn of the Advanced Materials and Device Sciences Department for assistance with the electronic transport measurements and A. Baca of the Compound Semiconductor Materials and Processes Department for assistance in fabrication of device structures for these measurements.

We thank Prof. S. Dumar from the Department of Physics and Liquid Crystal Institute at Kent State University for allowing us the use of the $18 \mathrm{KW}$ rotating anode $\mathrm{x}$-ray source, and Dr. Y. Shi, also from Kent State for assistance in setting up the reflectivity experiments and for use of the analysis routine.

The knowledge developed in this LDRD project was in part made possible by the close connection with several other Sandia programs, including earlier LDRDs, as well as a CRADA program with the Flat-Panel Display Division of Motorola Corporation. In particular, we wish to thank the following members of the Motorola FPDD team for many discussions concerning the emission properties of carbon films and their applications: J. Jaskie, B. Coll, A. Talin, E. Menu, P. von Allman and J. Song.

This LDRD project is directly responsible for developing a new collaboration with Profs. W. Milne and J. Robertson of Cambridge University for the study of a-C films, and in particular, their electron emission properties. 


\section{Contents}

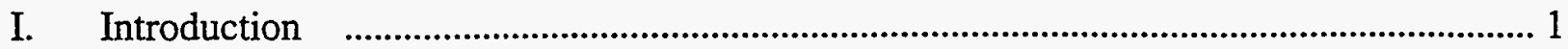

II. Raman Spectroscopy of Amorphous Carbon …................................................................ 3

III. Structural Characterization of the Thermal Evolution of Amorphous Carbon Films ..... 16

IV. The Electronic Transport Mechanism in Amorphous Carbon Films ….......................... 21

V. Electron Emission Induced Modifications in Amorphous Carbon …................................30

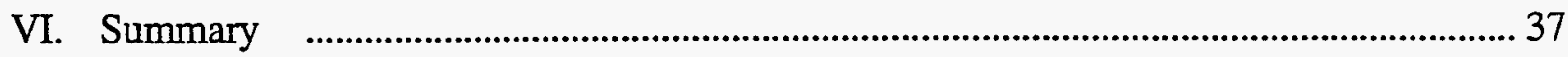

\section{Figures}

2.1 Raman spectra of forms of elemental carbon …............................................................. 4

2.2 Raman spectra vs. PLD growth energy density ….......................................................... 6

2.3 Raman spectra vs. a-C film thickness …................................................................. 7

2.4 Correlation of Raman spectra with film density _............................................................ 8

2.5 UV and visible Raman spectra of a-C film grown at $5 \mathrm{~J} / \mathrm{cm}^{2}$

2.6 Raman spectra of a-Si grown on a crystalline Si substrate ............................................ 11

2.7 Fractions of Raman spectra from various $C$ ring structures vs. growth energy $\quad . . . . . . . . . . .13$

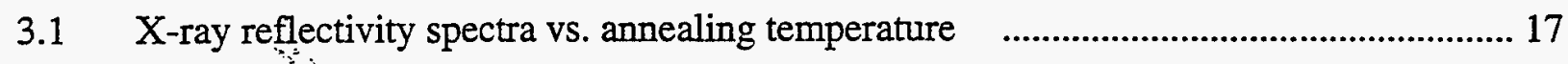

3.2 Overlay of reflectivity spectra from as-grown and $200^{\circ} \mathrm{C}$ annealed films

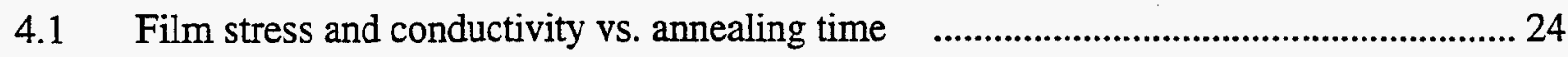

4.2 Electrical conductivity vs. change in a-C bonding via annealing .................................. 24

4.3 Activation energy for electrical transport …............................................................. 25

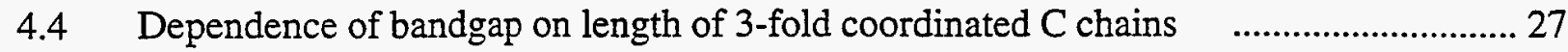

5.1 STM image of a-C film following emission preconditioning …................................. 32

5.2 STM and spatially-resolved EELS images of conditioned regions _............................. 33

5.3 Intermittent contact and surface potentiometry images of modified film $\ldots . . \ldots \ldots \ldots \ldots \ldots . . . . .35$ 


\section{Cold-Cathodes for Sensors and Vacuum Microelectronics}

\section{Introduction}

The major thrust of this project was to study the properties of amorphous carbon (a-C) films for the later development of new cold-cathode electron sources for vacuum microelectronics (VME) devices. These include intrinsically radiation-hard "vacuum tubes on a chip", as well as electron sources for miniature mass ionizers for advanced microsensors. There does not exist a fundamental understanding of why these a-C films are such good electron emitters. We combine carbon film growth and characterization for the study of carbon-based cold-cathode electron emission.

Existing emitter technologies use Spindt microtips fabricated from either silicon or molybdenum. The fabrication of these tips is both time-consuming and expensive. In addition, the high local electric fields generated during operation of emission tips can cause erosion of the tip surface, limiting its useful lifetime.

Amorphous carbon films, developed in part by Sandia, demonstrate both the required superb emission properties and compatibility with existing microelectronics fabrication. Use of these new materials for cold-cathode electron emission represent a leap-frog technology. Our challenge is to understand the emission mechanism and to utilize the knowledge for the eventual development of optimized materials for stable, robust device structures.

The amorphous-carbon bonding networks allow conformal coatings that are ultra-hard and chemically-resistant on most surfaces. These materials are deposited at room-temperature. Growth energetics control and determine the resulting bonding. Materials properties such as electrical conductivity, dielectric permittivity, optical absorption, and hardness are each tunable over a wide range.

VME devices are expected to offer a level of radiation hardness impossible to obtain in conventional solid-state microelectronics. VME device performance (high-voltage, high-power and high-speed) should be comparable to that of a vacuum tube, but on a size scale useful for integrated circuits. These simple VME devices could therefore be integrated into nuclear weapon and reactor environments that are too aggressive for any current or foreseeable alternative technology. 
Miniature ion mobility and mass spectrometers can be made by substituting the hot filament in the ionizer of conventional spectrometers with a cold-cathode emitter, simultaneously reducing both power and size requirements. In addition, the use of a cold-cathode electron source will eliminate molecular cracking and reactions associated with hot filaments that interfere with interpretation and quantitative analysis. This approach is an essential feature for monitoring waste streams where mixtures of complex organic molecules are common. In addition, miniaturization will permit operation at ambient pressures (several orders of magnitude higher than conventional mass spectrometers). These advances in miniaturization open up new classes of mass spectrometer applications for self-monitoring weapons and storage environments.

While a-C has demonstrated excellent emission properties, i.e., high emission currents at low applied voltages at room temperature, an understanding of the relationship between growth energetics, atomic bonding structures, materials properties and electron emission, does not exist. Amorphous- $C$ films are deposited at room temperature onto nearly any substrate material and have atomically smooth morphology. Pulsed-laser deposition (PLD) is used to grow a-C thin films. Film bonding structures are extensively studied using Raman spectroscopy and $\mathrm{x}$-ray reflectivity. Electrical transport measurements were made to better understand film conductivity. Electron emission properties were measured using scanning probe methods. 


\section{Raman Spectroscopy of Amorphous Carbon}

Amorphous carbon is an elemental form of carbon with low hydrogen content, which may be deposited in thin films by the impact of high energy carbon atoms or ions. It is structurally distinct from the more well-known elemental forms of carbon, diamond and graphite. It is distinct in physical and chemical properties from the material known as diamond-like carbon, a form which is also amorphous but which has a higher hydrogen content, typically near 40 atomic percent. Amorphous carbon also has distinctive Raman spectra, whose patterns depend, through resonance enhancement effects, not only on deposition conditions but also on the wavelength selected for Raman excitation. This section provides an overview of the Raman spectroscopy of amorphous carbon and describes how Raman spectral patterns correlate to film deposition conditions, physical properties and molecular level structure.

The elemental forms of carbon include a range of structures whose end members are diamond and graphite. In diamond the carbon atoms are four-coordinated and connected by bonds with $\sigma$-character (single bond order) in an $\mathrm{sp}^{3}$ (tetrahedral) configuration. Graphite is composed of stacked, planar sheets of six-membered rings of three-coordinated carbon. Graphitic carbon has conjugated single and double bonds in a ( $\pi$-bonded) $\mathrm{sp}^{2}$ configuration. The stacking of planar sheets of six-membered rings is integral to the structure of graphite and affects both its properties and its spectroscopic signature.

Carbon films deposited by the impact of high energy carbon atoms or ions, which are accelerated from a graphite target by pulsed laser ablation [1,2] or a filtered arc [3], can form an amorphous matrix with a complex molecular structure. First principles theoretical simulations of this amorphous carbon film (also known as amorphous tetrahedral carbon [4-6]) predict a rich variety of hybridized bonding which is not like that of diamond $\left(\mathrm{sp}^{3}\right)$ or graphite $\left(\mathrm{sp}^{2}\right)$ but includes three-fold coordinated and four-fold coordinated carbon atoms. For depositions utilizing higher energy excitation conditions, the four-coordinated carbon atoms are in the majority. Estimates of four-coordinated carbon comprising up to $80 \%$ of the total species have been made (see, e.g., [7]).

Raman spectra of elemental carbon reflect the differences in bonding of the different forms. Figure 2.1 shows the Raman spectra of crystalline diamond, an amorphous carbon film, nanocrystalline graphite and graphite with microscale to macroscale crystalline domains. These spectra were obtained using excitation at visible wavelengths. Diamond has a single Raman band in this frequency region, peaking at $1332 \mathrm{~cm}^{-1}$. Graphite has a Raman band due to a 
Brillouin-zone-center phonon at $1578 \mathrm{~cm}^{-1}$ (the "G" band) and a Raman band peaking near 1350 $\mathrm{cm}^{-1}$ (the " $\mathrm{D}$ " band), which is due to a zone-edge phonon that becomes symmetry-allowed in the reduced-symmetry environment at the edges of graphite crystals [8]. The Raman bands of graphite are enhanced in intensity with visible excitation by the interaction of electronic $\left(\pi \rightarrow \pi^{*}\right)$ transitions with vibrational transitions, such that Raman spectra of sub-micrometer thicknesses of graphite are easily obtained. Diamond Raman bands do not experience resonance enhancement with visible excitation, since vacuum ultraviolet wavelengths are required to access the $\left(\sigma \rightarrow \sigma^{*}\right)$ electronic transitions of diamond.

Graphite retains its structure of stacked planes of six-membered carbon rings even with domain sizes down to nanometer-scale dimensions, and the Raman spectra of "nanocrystalline" graphite show broadened bands and enhanced intensity in the " $D$ " band [9]. The double-banded structure shown in the Raman spectrum of Figure 2.1 is characteristic of graphite with nanoscale domains.

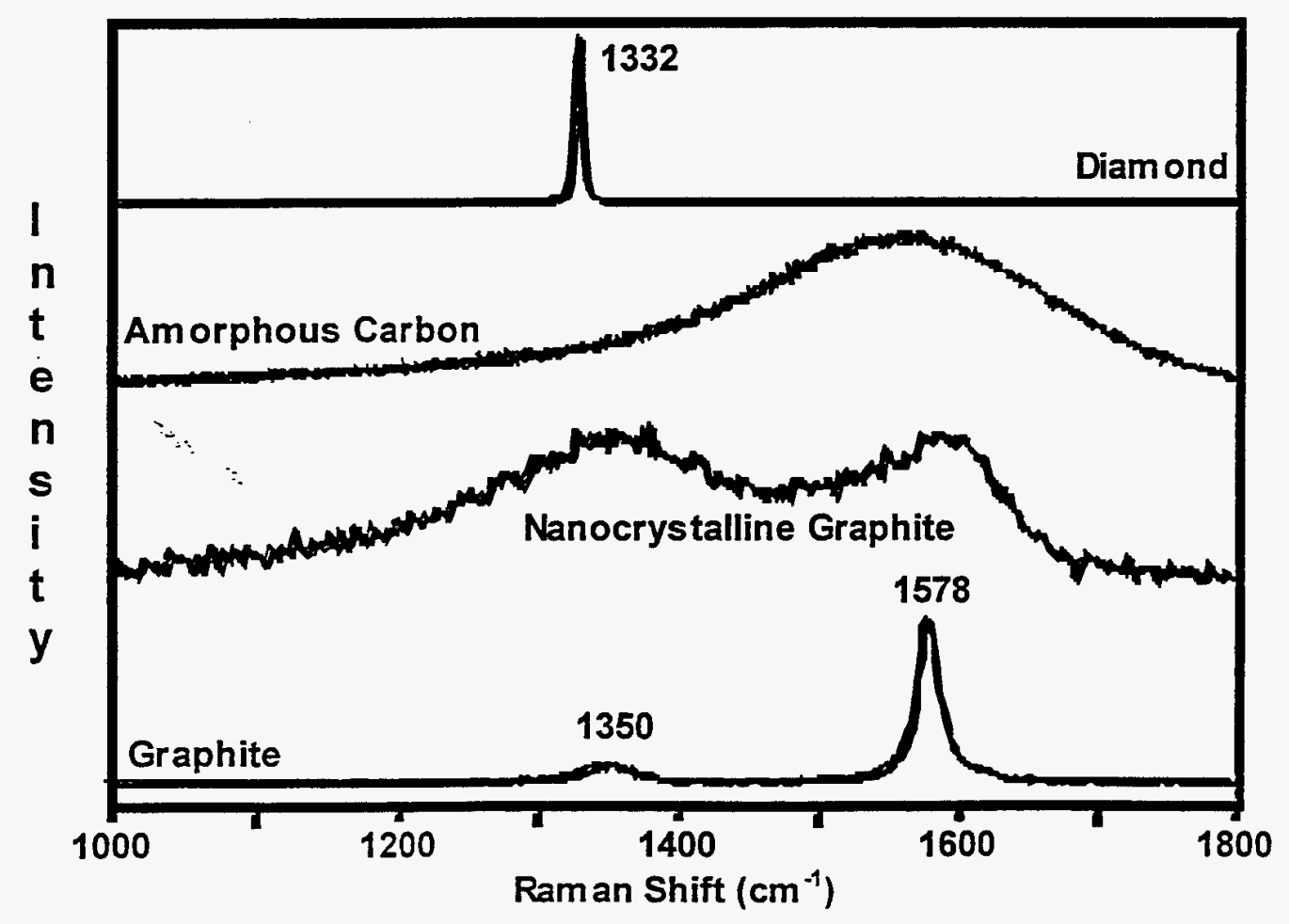

Figure 2.1. Raman spectra of forms of elemental carbon 
Vibrational bands from three-coordinated ( $\pi$-bonded) carbon structures dominate the Raman spectra of amorphous carbon when visible wavelengths are used for excitation. As with graphite, these three-coordinated carbon structures experience resonance enhancement of their Raman band intensities. However, the characteristic, two-band Raman pattern of graphite is generally not present in the Raman spectra of amorphous carbon (see Figure 2.1). Rather there is a broad band of complex shape, peaking from 1500 to $1570 \mathrm{~cm}^{-1}$, whose peak position and shape vary with deposition conditions. There appear to be a multiplicity of three-coordinated carbon structures contributing to the Raman spectra, whose distribution varies in relation to the properties of the amorphous carbon films.

There is less resonance enhancement of the three-coordinated carbon structures with ultraviolet excitation. Raman spectra of amorphous carbon films show enhanced intensity in a broad band peaking at 1100 to $1200 \mathrm{~cm}^{-1}$, which has been interpreted as being due to fourcoordinated carbon structures [7, 10]. Apparently, the reduction in intensity of the Raman bands due to three-coordinated carbon structures permits the observation of Raman bands due to fourcoordinated carbon structures. Thus, Raman with ultraviolet excitation is useful for detecting differences in the ratio of four-coordinated to three-coordinated carbon structures in amorphous carbon.

The carbon films whose spectra are displayed in this paper were deposited on silicon wafer substrates by pulsed $(248 \mathrm{~nm}$ ) laser ablation off a graphite target in vacuum [2]. Raman spectra were obtained using an argon ion laser for excitation (visible and near ultraviolet wavelengths). A triple spectrograph and charge-coupled-device detector were used to record the Raman spectra.

Figure 2.2 shows Raman spectra, excited with 514-nm laser light, of a series of amorphous carbon films, all approximately $100 \mathrm{~nm}$ thick, which were deposited with laser ablation energies from 5 to $125 \mathrm{~J} / \mathrm{cm}^{2}$. Higher deposition energies are believed to favor the formation of fourcoordinated carbon [11]. In addition to a broad carbon Raman band peaking between 1500 and $1600 \mathrm{~cm}^{-1}$, there is a flat-topped Raman feature between 900 and $1000 \mathrm{~cm}^{-1}$, which is a secondorder phonon band from the silicon substrate. The silicon Raman band increases in intensity with increasing deposition energy. The increase in silicon Raman band intensity implies that the films become more transparent as the deposition energy increases. The reason for this increase in transparency is believed to be a decrease in three-coordinated carbon structures with increasing deposition energy, resulting in less absorption of visible light by $\pi \rightarrow \pi^{*}$ electronic transitions. The carbon Raman band, which is due to structures incorporating three-coordinated 
carbon atoms, shifts to higher frequency and becomes narrower and more symmetric with increasing deposition energy. Its integrated intensity decreases as the proportion of threecoordinated carbon atoms decreases. Increasing deposition energy appears not only to reduce the overall proportion of three-coordinated carbon atoms but also to narrow the distribution of structures in which they reside, resulting in a smaller spread of vibrational energies.

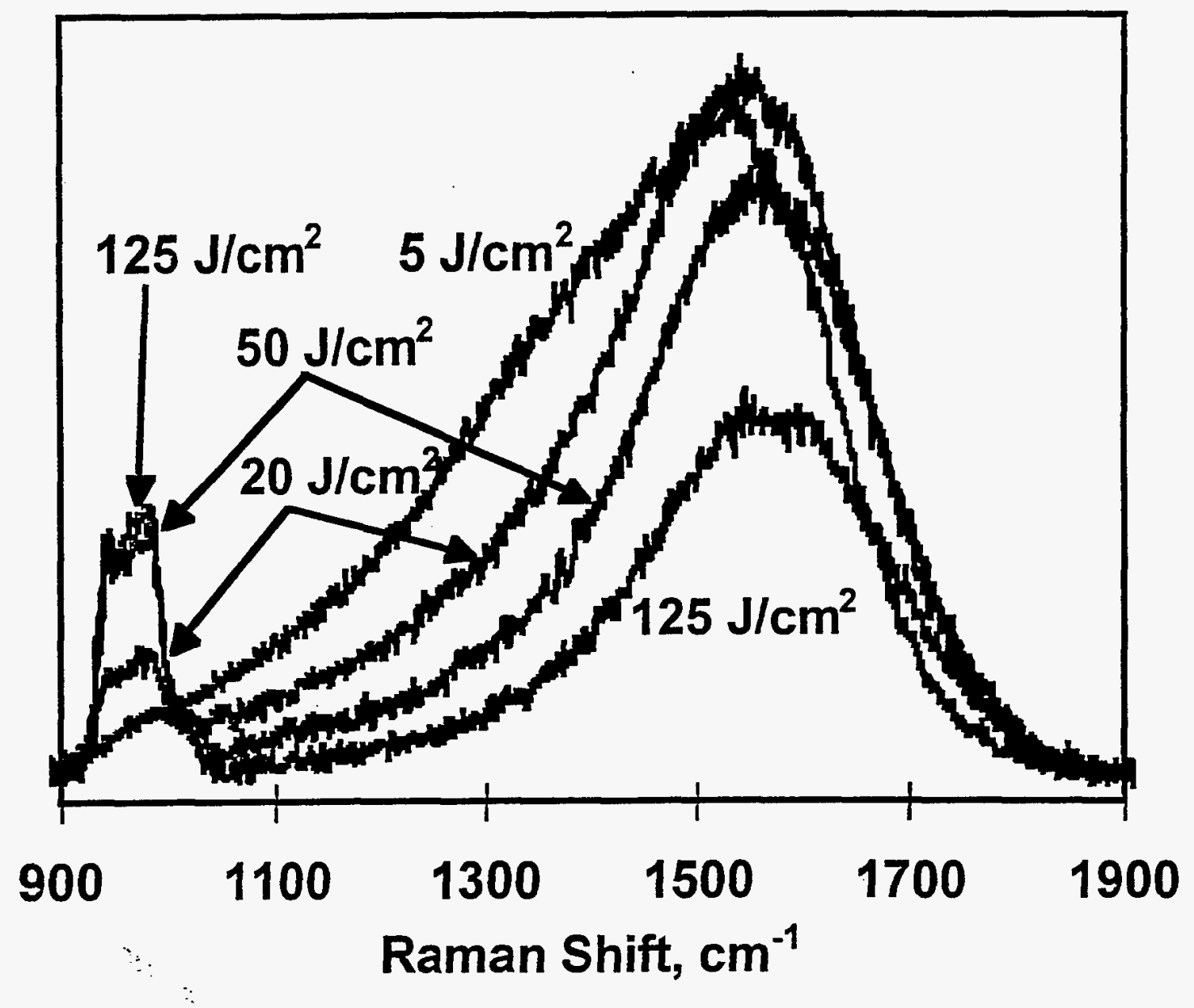

Figure 2.2: Raman spectra of amorphous carbon films deposited with laser energies as shown on the figure. The Raman excitation wavelength was $514 \mathrm{~nm}$.

Film thickness also affects the appearance of Raman spectra of amorphous carbon. In Figure 2.3 we show spectra of two films, both deposited at $44 \mathrm{~J} / \mathrm{cm}^{2}$, one of which was grown to a thickness of $7 \mathrm{~nm}$ and the other to a thickness of $200 \mathrm{~nm}$. The spectrum of the thicker film has the appearance of the films in Figure 2.2 grown at moderate-to-high deposition energies. The carbon Raman band is relatively narrow and symmetric and peaks at a relatively high frequency 
(about $1545 \mathrm{~cm}^{-1}$ ). Films deposited at $44 \mathrm{~J} / \mathrm{cm}^{2}$ and thicker than $50 \mathrm{~nm}$ give spectra like that of the 200 -nm thick film.

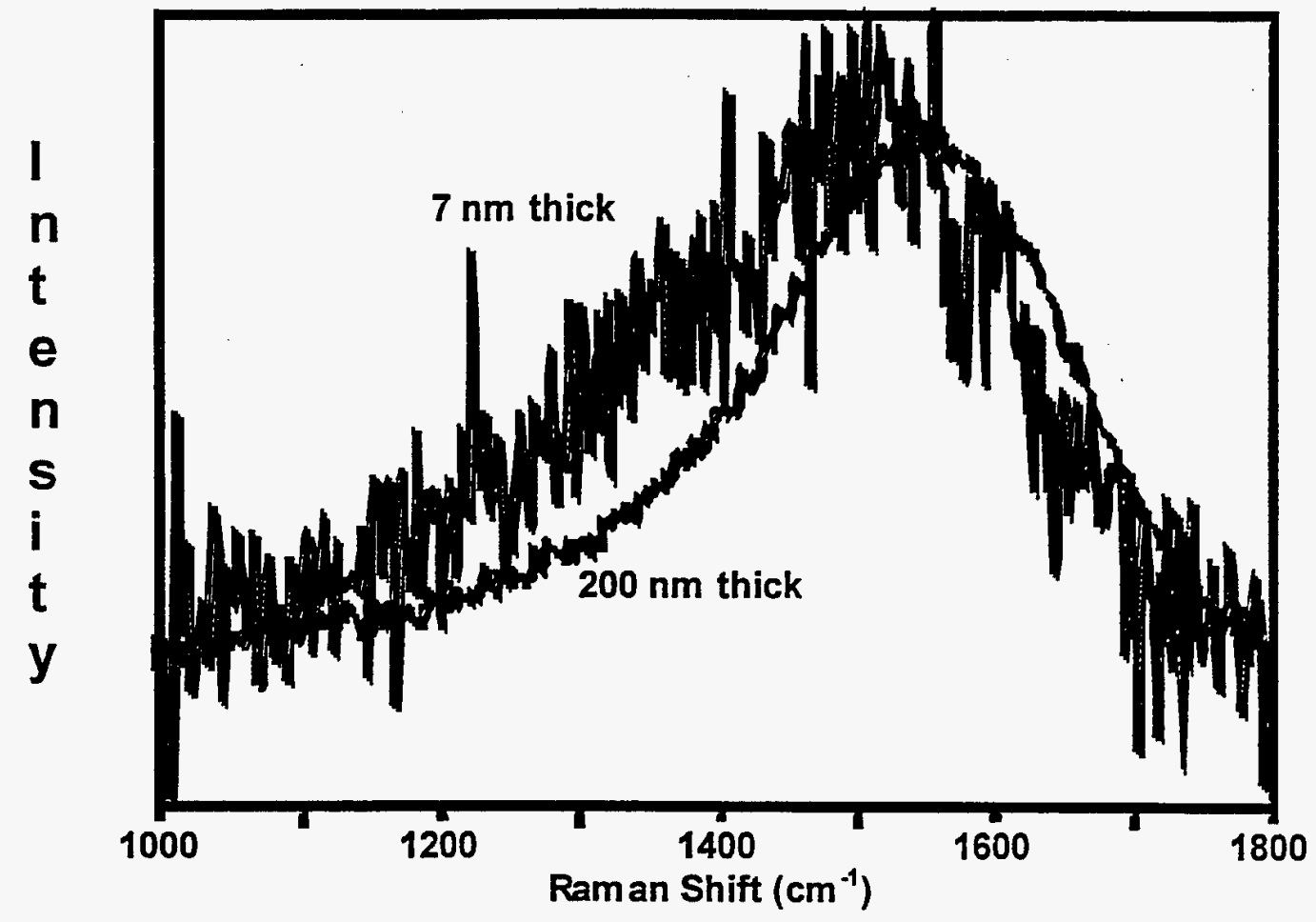

Figure 2.3: Raman spectra of amorphous carbon films deposited, at $44 \mathrm{~J} / \mathrm{cm}^{2}$, to thicknesses of 7 $\mathrm{nm}$ and $200 \mathrm{~nm}$. The Raman excitation wavelength was $514 \mathrm{~nm}$.

But the carbon Raman band of the thin $(7 \mathrm{~nm})$ film peaks at a significantly lower frequency $\left(1405 \mathrm{~cm}^{-1}\right)$ and is broadened asymmetrically to lower frequency, much like those carbon Raman bands deposited at lower energies (Figure 2.2). Clearly the 7-nm film is dominated by an interfacial layer which is different from the bulk of thicker films. The composition of the layer is not known for sure, but it may have a relatively high proportion of three-coordinated carbon atoms, as if it were deposited at a lower deposition energy. Alternately, the incorporation of silicon atoms could explain the downward shift in frequency of the carbon Raman band. Silicon atoms are more massive than carbon, and $\mathrm{Si}-\mathrm{C}$ bonds are less strong than $\mathrm{C}-\mathrm{C}$ bonds. Both higher mass and lower bond strength result in a lower vibrational frequency.

Although three-coordinated structures tend to be in the minority in carbon films, their Raman spectra reflect changes not only in their structures (Figure 2.2) but also in the physical properties of the film. In Figure 2.4 we plot densities of carbon films, as predicted by their Raman spectra, versus densities obtained by fitting glancing angle x-ray reflectivity curves. The 
carbon films were all about $100 \mathrm{~nm}$ thick. The densities of the films range from 2.2 to $3.0 \mathrm{~g} / \mathrm{cm}^{3}$, generally increasing with deposition energy. The density is believed to increase as the proportion of four-coordinated carbon in the films increases.

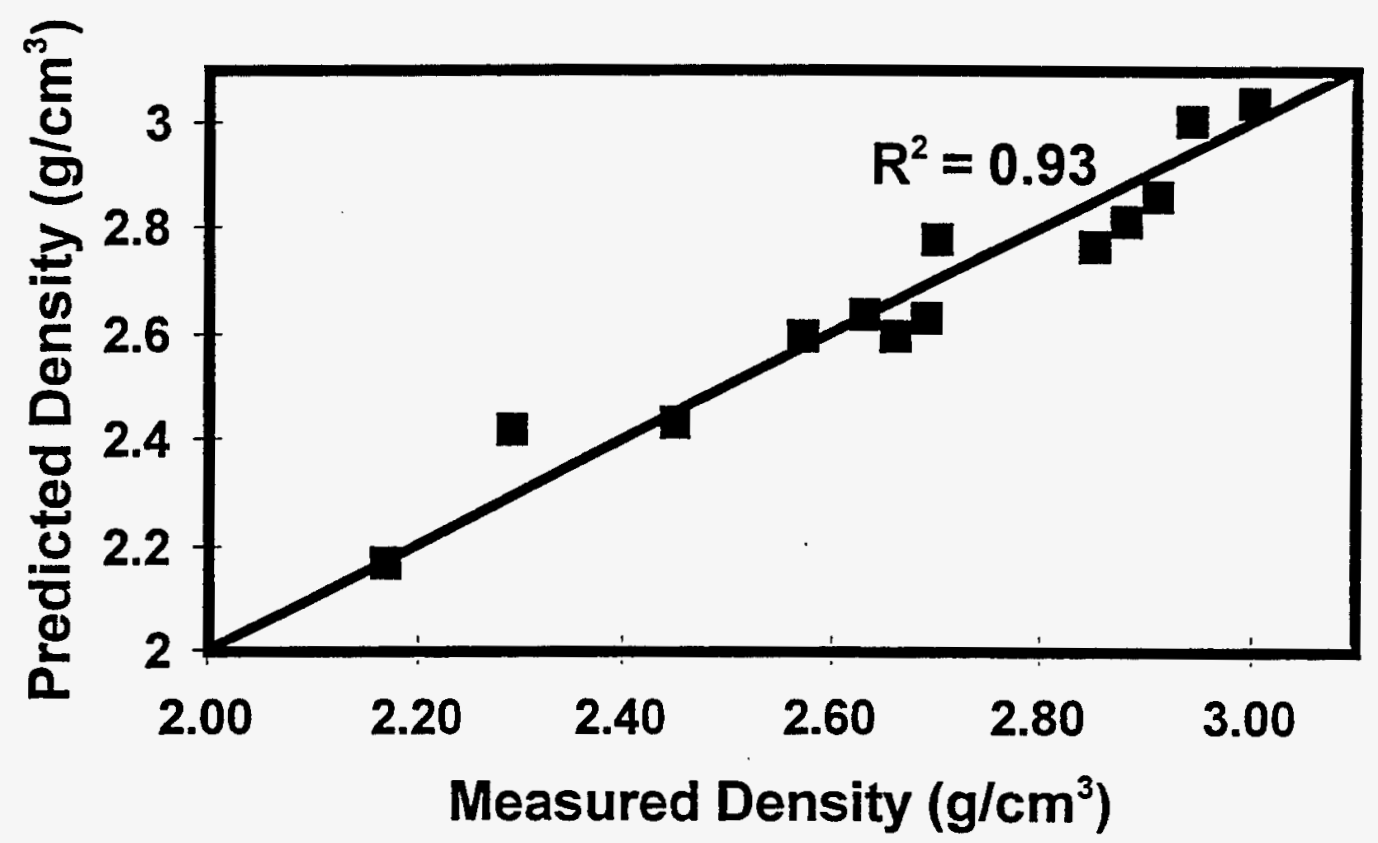

Figure 2.4: Density of carbon films predicted by Raman spectra versus measured by $\mathrm{x}$-ray diffraction. The prediction was done by multivariate partial least squares analysis.

The prediction of the densities was performed by multivariate partial least squares (PLS) analysis, which decomposes the Raman spectra into a set of components (like partial spectra) that are common to all the spectra and that correlate to the density. These components are used to reconstruct each Raman spectrum by summing the components with different scaling factors. The combination of spectral components and scaling factors forms a calibration file. The prediction also used cross validation. In cross validation one spectrum is excluded from the set of samples, and a calibration is constructed using the remaining spectra in the set and the measured densities. This "partial" calibration is then used to predict the density of the excluded sample from its Raman spectrum. The process is repeated for all the samples, resulting in a predicted density for each sample. If we plot predicted versus measured densities for each sample in the set (Figure 2.4), the result is ideally a line with a slope of 1, passing through the zero intercept, and with all the data points on the line. The correlation coefficient $\left(\mathrm{R}^{2}\right.$, which should be close to 1.00 ) measures how much the points deviate from the ideal line. The $R^{2}=0.93$ 
for the data in Figure 2.4 indicates a high correlation between density and the Raman spectra of three-coordinated carbons. A higher correlation $\left(\mathrm{R}^{2}=0.98\right)$ was obtained between Raman spectra of the three-coordinated carbon and the as-deposited stress in the film. Stress increases with deposition energy and is believed to also be related to proportion of four-coordinated carbon [11]. Less error is expected in the measurement of the stress than in the density measurement.

Raman spectra obtained with visible excitation wavelengths show changes in the transparency of the film and the distribution of structures incorporating three-coordinated carbon atoms as function of deposition energy. The carbon Raman band patterns from structures incorporating three-coordinated carbon atoms correlate to the density and the stress in the films. Thus, the distribution of structures incorporating three-coordinated carbon atoms reflects, at least to some extent, the overall structure and properties of the carbon film, and their Raman spectra is a useful characterization tool.

\section{RAMAN WITH ULTRAVIOLET EXCITATION}

Shifting the Raman excitation wavelength to ultraviolet wavelengths reduces the resonant enhancement associated with $\pi \rightarrow \pi^{*}$ electronic transitions and decreases the extent to which structures incorporating three-coordinated carbon atoms dominate the Raman spectra. Raman experiments utilizing deep ultraviolet $(244 \mathrm{~nm})$ excitation have shown the appearance, in the Raman spectra, of a broad band, peaking at 1100 to $1200 \mathrm{~cm}^{-1}$, whose intensity correlates with the proportion of four-coordinated carbon atoms in the films $[7,10]$. It is believed that the reduction in the intensity of the resonantly enhanced Raman bands from structures incorporating three-coordinated carbon atoms permits the observation of Raman bands due to four-coordinated carbon atoms. It is not yet clear whether the $\sigma \rightarrow \sigma^{*}$ electronic transitions of the four-coordinated carbon atoms extend far enough out of the vacuum ultraviolet to result in significant resonance enhancement with wavelengths above $200 \mathrm{~nm}$.

We have recently developed a capability for ultraviolet Raman and have obtained a Raman spectrum of a carbon film using near ultraviolet $(364 \mathrm{~nm}$ ) excitation (see Figure 2.5). Compared to a Raman spectrum of the same film obtained with visible $(514 \mathrm{~nm})$ excitation, the nearultraviolet-excited spectrum shows an additional, broad Raman band extending from $900-1400$ $\mathrm{cm}^{-1}$. Also, there is a shift, with ultraviolet excitation, of the three-coordinated carbon band peaking near $1600 \mathrm{~cm}^{-1}$ to higher frequency. Such shifts have been previously reported [7]. These preliminary results suggest that even at this (near) ultraviolet wavelength, suppression of resonance enhancement of the Raman bands from structures incorporating three-coordinated 
carbon atoms is sufficient to permit observation of a Raman band from four-coordinated carbon atoms.

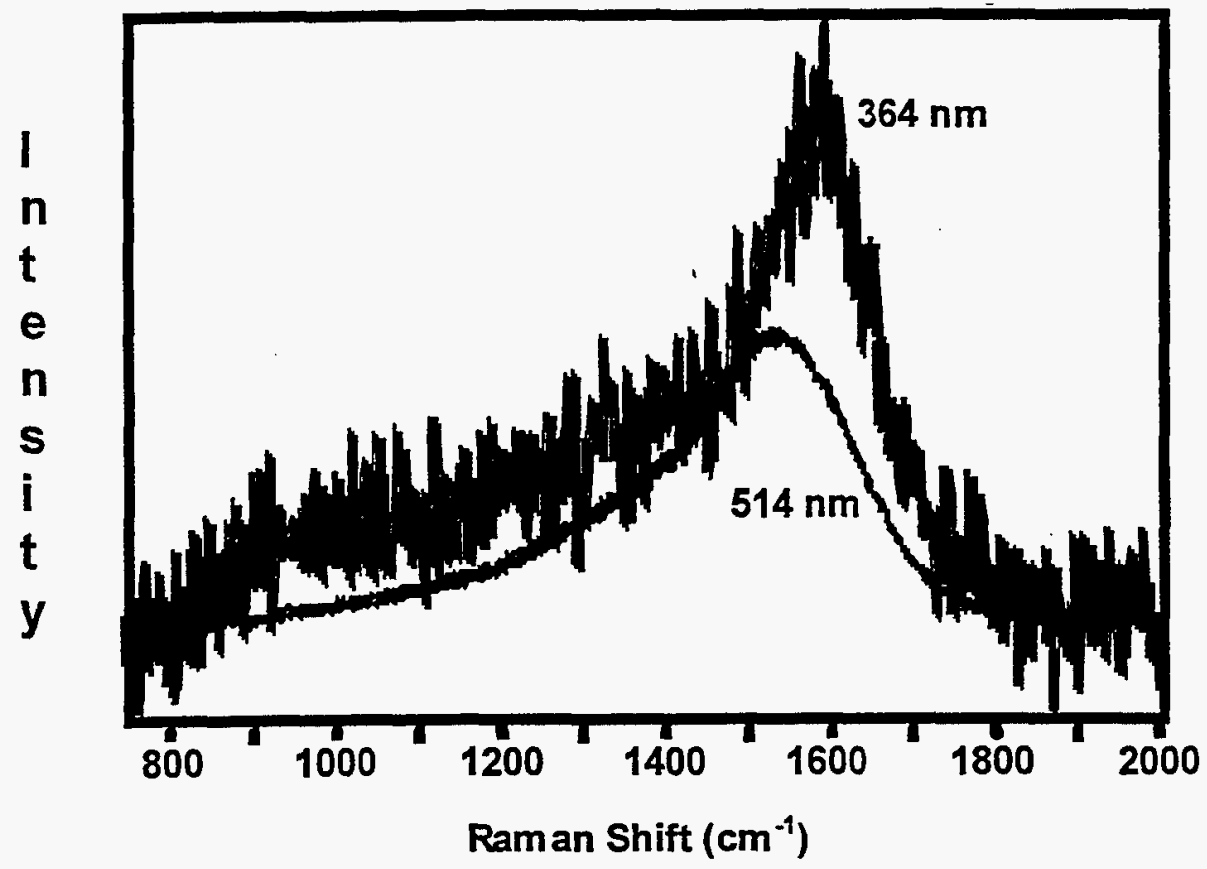

Figure 2.5: Raman spectra of a carbon film deposited at $5 \mathrm{~J} / \mathrm{cm}^{2}$, with Raman excitation at 364 $\mathrm{nm}$ and $514 \mathrm{~nm}$ as indicated in the figure. Intensities have been scaled.

Using ultraviolet excitation it is possible to record a Raman feature (the band peaking at 1100 to $1200 \mathrm{~cm}^{-1}$ ) which is associated with four coordinated atoms in amorphous carbon films. In the spectra reported thus far, this feature does not appear to shift in peak frequency or change in shape to the same extent as the Raman bands from to three-coordinated carbon structures. However, this Raman band provides a means, through its intensity changes, of directly monitoring changes in the proportion of four-coordinated carbon atoms.

\section{IMPLICATIONS OF RAMAN SPECTRA FOR CARBON FILM STRUCTURE}

\section{Four-Fold Coordinated Carbon}

Ultraviolet-excited Raman spectra include a broad band peaking at 1100 to $1200 \mathrm{~cm}^{-1}$, which has been associated with four-coordinated carbon. No definitive experiment has been performed to date to define the structure of the matrix component composed of four-coordinated carbon, but enough is known to suggest its molecular structure. First of all, it is not a crystalline (or nanocrystalline) form of diamond. Raman spectra of "cluster diamond", a form of crystalline 
diamond produced by explosive detonation and with a crystal size of about five nanometers, have been reported [12]. The spectra show a diamond band peaking near $1322 \mathrm{~cm}^{-1}$ and about 30 $\mathrm{cm}^{-1}$ in bandwidth, not a very broad band peaking near $1200 \mathrm{~cm}^{-1}$ which has a bandwidth of several hundred inverse centimeters.

A more likely possibility is that the four-coordinated carbon has the structure of an amorphous form of diamond. Here there is an analogy to the silicon system. The Raman band of crystalline silicon peaks near $520 \mathrm{~cm}^{-1}$ with a narrow $\left(<5 \mathrm{~cm}^{-1}\right)$ linewidth, but its amorphous analog peaks at $465-480 \mathrm{~cm}^{-1}$ with a linewidth of 60 to $100 \mathrm{~cm}^{-1}$ [13]. A Raman spectrum of an amorphous silicon film on a crystalline silicon substrate is shown in Figure 2.6.

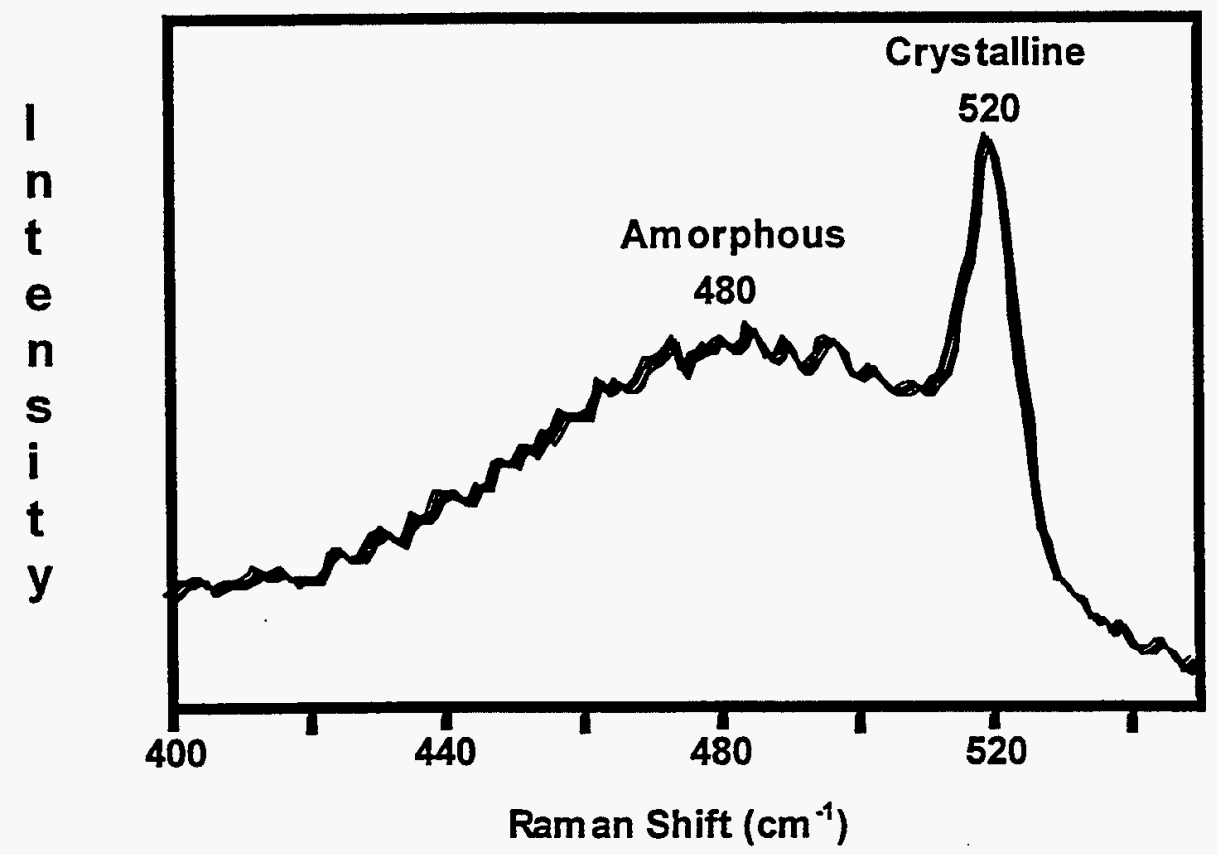

Figure 2.6: Raman spectrum of a film of amorphous silicon over a crystalline silicon substrate (514 nm excitation)

The Raman peak in silicon shifts downward from near $520 \mathrm{~cm}^{-1}$ to the vicinity of $480 \mathrm{~cm}^{-1}$ with a transition from a crystalline to an amorphous matrix. The decrease in frequency is due to an increase in bond lengths in the amorphous state. A proportional shift from the crystalline diamond peak $\left(1332 \mathrm{~cm}^{-1}\right)$ would result in an amorphous diamond band peaking near $1230 \mathrm{~cm}^{-1}$. Thus, the Raman band which is observed in ultraviolet-excited Raman spectra of amorphous carbon films and which increases in intensity with the amount of four-coordinated carbon is consistent with the Raman feature which would be expected from amorphous diamond. 


\section{Three-Fold Coordinated Carbon}

The complex changes in the Raman spectra of amorphous carbon films as a function of deposition conditions (see Figure 2.2) when the spectra are excited by visible wavelengths suggest that the structures incorporating three-coordinated carbon atoms may be large and complex. One possibility is that the three-coordinated carbon atoms are assembled into chains which have conjugated $\pi$-bonding. This possibility is supported by theoretical simulation $[4,6]$ and measurements of electronic transport [14], which suggest an average chain length of 13 atoms. Polyvinyl chloride (PVC) is a polymer which spontaneously generates conjugated polyene chains when it degrades by evolution of hydrogen chloride:

$$
\left(\mathrm{CH}_{2}-\mathrm{CHCl}\right)_{\mathrm{n}} \rightarrow(\mathrm{CH}=\mathrm{CH})_{\mathrm{n}}+\mathrm{HCl}
$$

Raman spectra of degraded PVC show resonance enhancement, with green excitation wavelengths, of bands from the conjugated chains when the chains reach about 17 polyene units in length ( $\mathrm{n}=17$, or 34 carbon atoms [15]). Two relatively narrow (about $30 \mathrm{~cm}^{-1}$ linewidth), approximately equal intensity, Raman bands appear resonantly enhanced in the spectra of degraded PVC. These bands peak about 1100 and $1500 \mathrm{~cm}^{-1}$. The Raman band pattern of PVC, with relatively narrow bands of equal intensity peaking near $1100 \mathrm{~cm}^{-1}$ and $1500 \mathrm{~cm}^{-1}$, cannot account for the Raman spectra of Figure 2.2, whose broad features peak from $1535 \mathrm{~cm}^{-1}$ to 1570 $\mathrm{cm}^{-1}$. We recognize that the Raman spectra in the PVC polyene units may be affected by the presence of hydrogen. However, even the Raman spectra of hydrogen-rich diamond-like (amorphous) carbon more closely resembles the Raman spectra of the low-deposition-energy films of Figure 2.2 than that of degraded PVC. It is probable that polyene-like chains exist in amorphous carbon films, but they don't appear to be the primary source of the amorphous carbon Raman bands.

Another possible set of structures incorporating three-coordinated carbon atoms include sheets made up of $\pi$-bonded rings composed of five, six or seven carbon atoms. Fullerenes and nanotubes [16] include these types of rings, with the six-membered components forming flat sheets and the five- and seven-membered components providing curvature. These complex structures may include tens to hundreds of carbon atoms in sheets or ribbons, which may be too large to model with simulation protocols that use $100-300$ atoms per unit cell [4-6]. While no experimental confirmation of the presence of $\pi$-bonded rings exists, theoretical calculations [17] have predicted the Raman frequencies of five - to seven-membered, $\pi$-bonded carbon rings. Using a vibrational mode of $E_{2 g}$ symmetry peaking at $1569 \mathrm{~cm}^{-1}$ to represent six-membered rings, 
plus modes of $A_{1}$. symmetry at $1444 \mathrm{~cm}^{-1}$ (five-membered rings) and $1303 \mathrm{~cm}^{-1}$ (sevenmembered rings) and a small contribution from an $E_{2}$, mode at $1100 \mathrm{~cm}^{-1}$ (five-membered rings), it is possible to reconstruct amorphous carbon Raman spectra over the range of deposition energies shown in Figure 2.2. Our fitting procedure used as adjustable parameters the intensities of the vibrational modes, with linewidths of 200 to $400 \mathrm{~cm}^{-1}$. Normalized integrated intensities of the calculated bands, fitted to spectra of amorphous carbon films deposited with energies from $5 \mathrm{~J} / \mathrm{cm}^{2}$ to $125 \mathrm{~J} / \mathrm{cm}^{2}$ (see Figure 2.2), are plotted in Figure 2.7 .

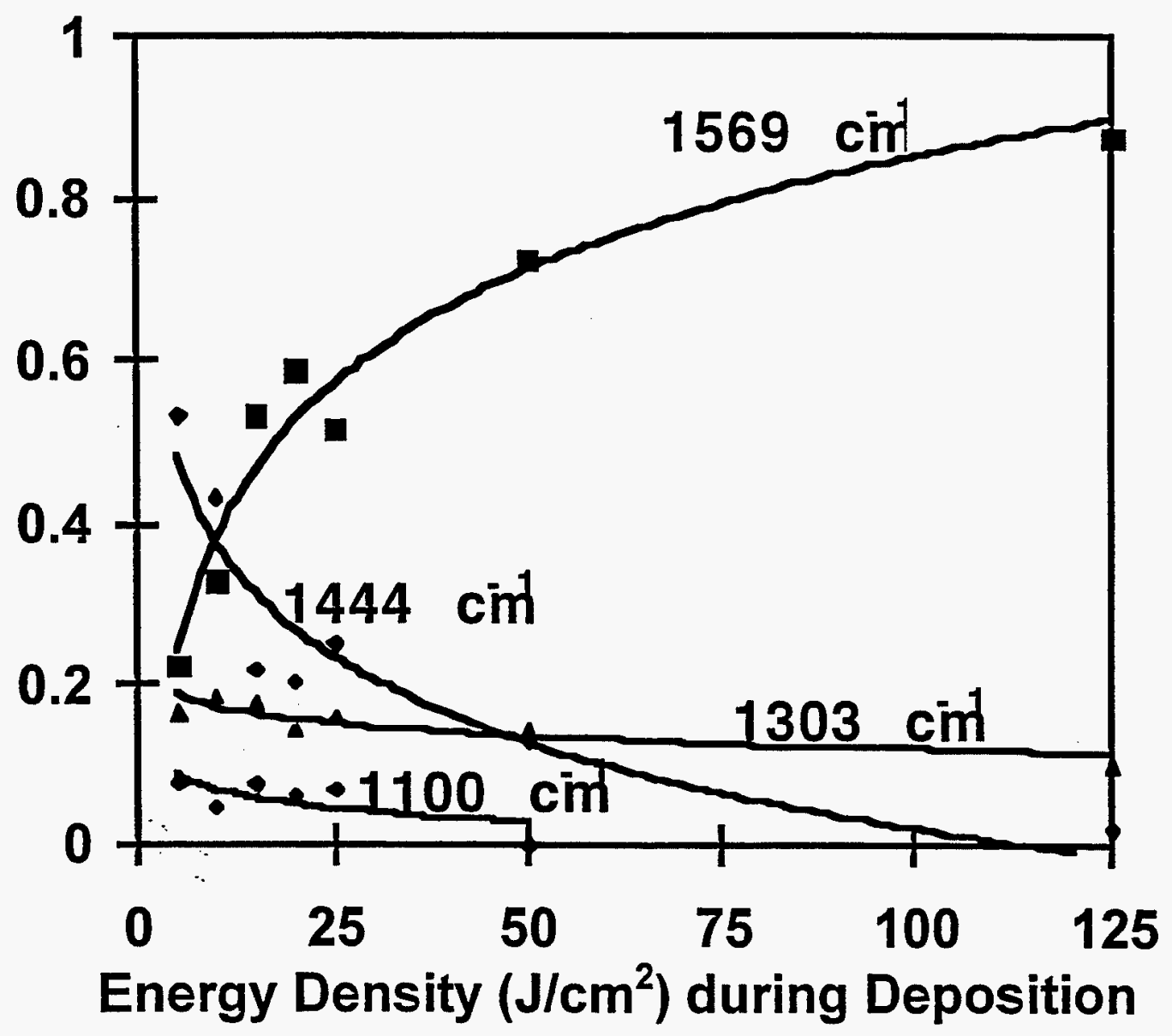

Figure 2.7: Normalized integrated intensities of the calculated vibrational modes fitted to Raman spectra of amorphous carbon films deposited at $5-125 \mathrm{~J} / \mathrm{cm}^{2}$. Squares - sixmembered rings $\left(1569 \mathrm{~cm}^{-1}\right)$; diamonds - five-membered rings $\left(1100 \mathrm{~cm}^{-1}\right.$ and $\left.1444 \mathrm{~cm}^{-1}\right)$; and triangles - seven-membered rings $\left(1303 \mathrm{~cm}^{-1}\right)$. 
The results of the fitting procedure, as shown in Figure 2.7, indicate that, as the deposition energy increases, so does the proportion of six-membered rings at the expense of five- and seven-membered rings. Since the overall proportion of structures incorporating threecoordinated carbon atoms also decreases with increasing deposition energy, it appears that flat sheets of six-membered carbon rings are favored when the overall proportion of threecoordinated carbon atoms is low, and the three-coordinated carbon structures are likely to be smaller and more isolated. At low deposition energies and, consequently, a higher proportion of three-coordinated carbon, the larger and more densely packed three-coordinated carbon structures tend to incorporate more five- and seven-membered rings, which impart curvature to their surfaces. One can rationalize this behavior by considering the alternative to an increase in the proportion of five- and seven-membered rings with increasing three-coordinated carbon. Sheets of (flat) six-membered carbon rings, present in high density, can be expected to stack, forming graphite-like structures with a distinctive Raman band pattern (Figure 2.1), which has not been observed in these amorphous carbon films.

Because of resonance enhancement effects, the Raman band pattern obtained from amorphous carbon films depends on the laser excitation wavelength. Visible excitation wavelengths resonantly enhance structures incorporating $\pi$-bonded, three-coordinated carbon atoms, because their $\pi \rightarrow \pi^{*}$ transitions occur at visible wavelengths. Four-coordinated carbon structures have electronic transitions $\left(\sigma \rightarrow \sigma^{*}\right)$ in the far (vacuum) ultraviolet, and their Raman bands do not experience resonance enhancement with visible excitation. Bands from threecoordinated carbon structures dominate the Raman spectra with visible excitation. With ultraviolet excitation the enhancement of Raman bands from three-coordinated structures is not as pronounced, and a broad band peaking at 1100 to $1200 \mathrm{~cm}^{-1}$, which is due to four-coordinated carbon, becomes observable. The peak position and breadth of this band are consistent with an amorphous analog of diamond, and this Raman band is useful in following the relative proportion of four-coordinated versus three-coordinated carbon. The Raman bands of threecoordinated carbon structures, as obtained with visible excitation, have broad and complex patterns, which vary with deposition conditions. This variation in the Raman band patterns correlates to physical properties of the carbon film, such as density and internal stress, and it is apparently related to changes in the distribution of three-coordinated carbon structures. Calculated Raman spectra of small, $\pi$-bonded carbon rings reproduce the experimental data well, but conjugated chain structures may also be contributing to the observed Raman features.

Raman spectra of amorphous carbon are rich in information, much of which we do not yet know how to interpret. Definitive experiments which will completely characterize the structure of amorphous carbon have yet to be carried out. 


\section{References}

1. Xiong, Y. Y. Wang and R. P. H. Chang, Phys. Rev. B 48, p. 8016 (1993).

2. T. A. Friedmann, K. F. McCarty, J. C. Barbour, M. P. Siegal and D. C. Dibble, Appl. Phys. Lett. 68, 1643 (1996).

3. J. Robertson, Prog. Solid State Chem. 21, p. 199 (1991).

4. N. A. Marks, D. R. McKenzie, B. A. Pailthorpe, M. Bernasconi and M. Parrinello, Phys. Rev. Lett. 76, p. 768 (1996).

5. S. Nelson, E. B. Stechel, A. F. Wright, S. J. Plimpton, P. A. Schultz and M. P. Sears, Phys. Rev. B 52, p. 9354 (1995).

6. P. A. Schultz and E. B. Stechel, Phys. Rev. B (in press).

7. W. R. Gilkes, H. S. Sands, D. N. Batchelder, J. Robertson and W. I: Milne, Appl. Phys. Lett. 70, p.1980 (1997).

8. Y. Wang, D. C. Alsmeyer and R. L. McCreery, Chem. Mater. 2, p.557 (1990).

9. F. Tuinstra and J. L. Koenig, J. Chem. Phys. 53, p. 1126 (1970).

10. V. I. Merkulov, J. S. Lannin, C. H. Munro, S. A. Asher, V. S. Veerasamy and W. I. Milne, Phys. Rev. Lett. 78, 4869 (1997).

11. J. P. Sullivan, T. A. Friedmann and A.G. Baca, J. Electr. Mat. 26, p.1021 (1996).

12. M. Yoshikawa, Y. Mori, H. Obata, M. Maegawa, G. Katagiri, H. Ishida and A. Ishitani, Appl. Phys. Lett. 67, p.694 (1995).

13. F. H. Pollak, Test and Measurement World, p. 2 (May, 1985).

14. J. P. Sullivan and T. A. Friedmann, in Proc. First International Specialist Meeting on Amorphous Carbon, (World Scientific Publishing, Singapore, 1997), Cambridge, U. K., 1997.

15. W.F. Maddams, American Laboratory, p.59 (March, 1986).

16. See the cover and articles of the MRS bulletin, November, 1994.

17. T. E. Doyle and J. R. Dennison, Phys. Rev. B. 51, p. 196 (1995). 


\section{Structural Characterization of the Thermal Evolution of Amorphous Carbon Films}

Amorphous carbon (a-C) films are of interest due to their high thermal stability, good tribological properties and low-electric field cold-cathode emission. A significant drawback for the use of these films in electronic applications is the several GPa high residual compressive stress present in as-grown films. We reported recently that thermal annealing to $600{ }^{\circ} \mathrm{C}$ of a-C films grown by pulsed laser deposition (PLD) reduces the as-grown film stress to negligible levels and that some stress reduction occurs at annealing temperatures as low as $100^{\circ} \mathrm{C} .[1]$

The above study also showed that stress reduction occurs without changing significantly the optical transparency or the electrical resistivity of the films. Other work on amorphous carbon $(\mathrm{a}-\mathrm{C})$ films indicated that a-C density decreases with increasing annealing temperature.[2] We present the results of a structural evolution on a-tC films on Si(100) prepared using PLD and subsequently annealed for two hours at temperatures ranging from room temperature to $600{ }^{\circ} \mathrm{C}$. The evolution is studied using a combination of $\mathrm{x}$-ray reflectivity and small angle scattering. The films exhibit a decrease in density with increasing annealing temperature, similar to the one exhibited by a-C films. In addition, the films exhibit an evolution in both surface and interface roughness with annealing temperature. A small angle scattering signal, observed as a function of deposition energetics in similarly prepared samples changes position as a function of temperature.[3,4]

We use PLD to grow a-C films onto $\mathrm{Si}(100)$ wafers from a rotating pyrolytic graphite target with $248 \mathrm{~nm}$ excimer pulsed-laser radiation $(\mathrm{KrF})$. The details of the deposition process are similar to those described elsewhere.[5] Films nominally $90 \mathrm{~nm}$ thick are grown in a vacuum chamber with base pressure $<10^{-7}$ Torr with laser energy density $=45 \mathrm{~J} / \mathrm{cm}^{2}$. The Si substrates are precleaned with acetone, methanol, and a brief immersion in dilute HF prior to deposition. Post-deposition thermal annealing is performed using a commercial rapid thermal processing system in 630 Torr Ar gas (atmospheric pressure in Albuquerque, NM) in a fused silica chamber with external heating lamps. Similar a-C films are thermally treated at temperatures ranging from 200 to $600^{\circ} \mathrm{C}$ for two hours each. Two hour anneals were found to saturate the stress relief for a given temperature.[1]

The $\mathrm{x}$-ray studies were performed using the $\mathrm{Cu} \mathrm{K}_{\alpha 1}$ radiation from an $18 \mathrm{~kW}$ rotating

anode source with a resolution of $0.0001 \AA^{-1}$. The resulting reflectivity curves were fit to the Fresnel interference curve for a film of finite thickness $d$. The small angle signals were fit to 
Gaussian curves. The resulting fit allows us to extract information on the density, the roughness and the thickness of the films, as well as the position evolution of the small angle signal.

Figure 3.1 shows the $\mathrm{x}$-ray reflectivity data obtained from an as-grown a-C film as well as films annealed at $200,300,400,500$ and $600^{\circ} \mathrm{C}$ respectively. In addition to the characteristic reflectivity curve, all the films exhibit a diffraction peak about $2 \theta=2^{\circ}$, which changes position as a function of annealing temperature. Figure 3.2 shows the superposition of the reflectivity from the as-grown sample and from the sample annealed at $200^{\circ} \mathrm{C}$. We notice an increase in the near surface roughness in the sample annealed at $200^{\circ} \mathrm{C}$.

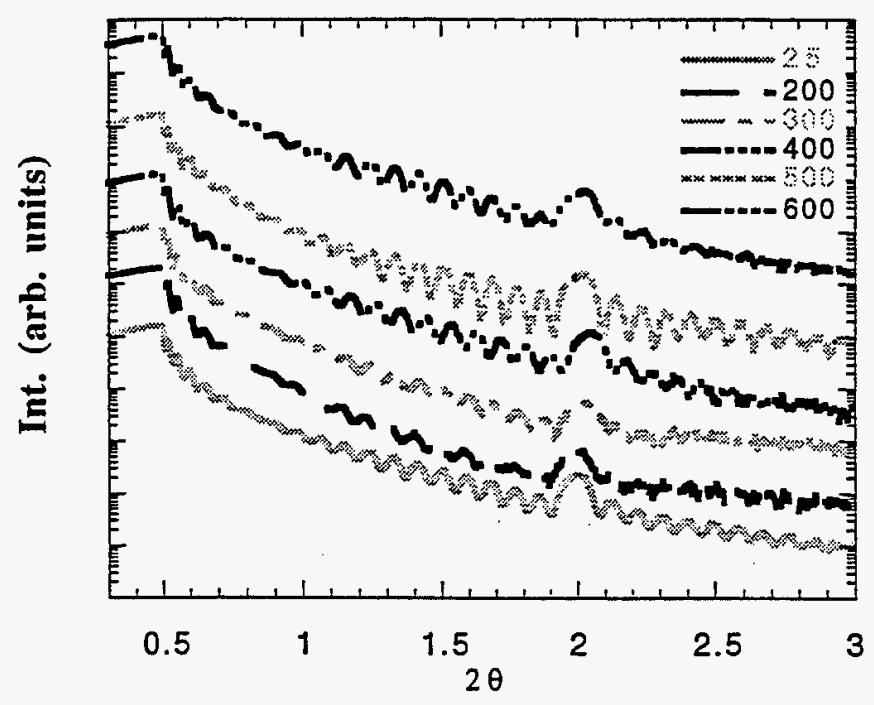

Figure 3.1: $\mathrm{X}$-ray reflectivity curves obtained from the temperature annealed samples. The curves have been displaced along the ordinate for clarity.

The reflectivity curves are fit to a function,

$$
I=f\left(R_{j}(\theta), \exp \left(-\sigma_{j}^{2} q^{2}\right)\right)+\Sigma A_{i} * G\left(q_{i}\right),
$$

where $R_{j}(\theta)$ is the Fresnel reflectivity curve of a film with two interfaces, $\sigma_{j}$ is the roughness of the interfaces, $q$ is the scattering wavevector, $A_{i}$ are constants and $G\left(q_{i}\right)$ is a Gaussian function of width $\Delta q$.[6] The function $f\left(R_{j}(\theta), \exp \left(-\sigma_{j}^{2} q^{2}\right)\right)$ describes the reflectivity of a number of multilayers. All films are fit to two Gaussians, centered about $0.05 \AA^{-1}$, and $0.14 \AA^{-1}$ respectively. The films annealed at $500^{\circ} \mathrm{C}$ and $600^{\circ} \mathrm{C}$ were also fit to a multilayer reflectivity curve. Table I 
shows the values obtained for the density $\rho$, the film roughness $\sigma$, and the position of the small angle peak obtained from the fits.

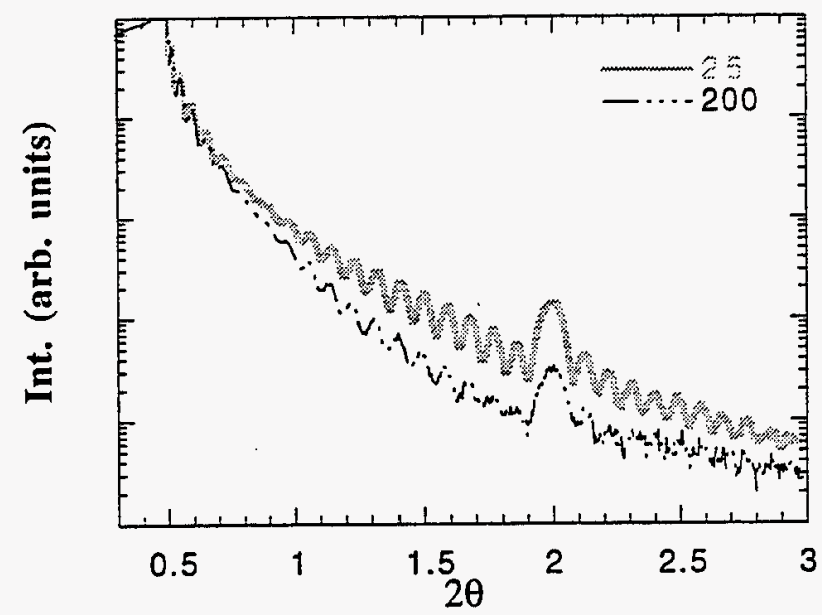

Figure 3.2: Comparison of the reflectivity curves for the as-grown film and the film annealed at $200^{\circ} \mathrm{C}$. The faster decay of the curve for the $200^{\circ} \mathrm{C}$ film indicates increased roughness.

Table I shows that the roughness of the film increases from being atomically smooth [7] for the as-grown film to a magnitude of approximately $1 \mathrm{~nm}$ at $200{ }^{\circ} \mathrm{C}$, and then decreasing monotonically above this temperature. Atomic smoothness is recovered by $600^{\circ} \mathrm{C}$.

Table I: Structural properties of a-C films as a function of annealing temperature, obtained from the fit to equation 1 .

$\underline{\mathrm{T}_{2}\left({ }^{\circ} \mathrm{C}\right) \text { densitv }\left(\mathrm{g} / \mathrm{cm}^{3}\right)}$ roughness $(\AA)$ small angle feature spacing $(\AA)$

$\begin{array}{llll}25 & 3.01 & 0.0 & 44.19 \\ 200 & 3.00 & 9.0 & 43.91 \\ 300 & 2.95 & 6.0 & 43.56 \\ 400 & 2.86 & 2.0 & 43.12 \\ 500 & 2.81 & 2.0 & 43.61 \\ 600 & 2.79 & 0.0 & 43.78\end{array}$


The shape of the reflectivity curve is also affected by the presence of a small scattering peak near $2 \theta=2^{\circ}\left(q=0.14 \AA^{-1}\right)$, present in all samples. The feature is related possibly to a quasi-periodic array of scattering sites with a spacing in the order of $4 \mathrm{~nm}$. This feature may have its maximum slightly off the reflectivity axis. The spacing corresponding to this feature changes as a function of annealing temperature, as observed in Figure 3.1 and Table I. It achieves its smallest value at $400{ }^{\circ} \mathrm{C}$, the same temperature at which the magnitude of the changes in resistivity and density decrease. The decrease in the spacing value correlates with the increase in film roughness observed in these films: the increase in spacing observed above 400 ${ }^{\circ} \mathrm{C}$ coincides with the recovery of atomic smoothness in the samples. This feature has been observed previously in the study of a-C as a function of PLD growth energetics.[3] A report on the evolution of this feature as a function of these growth energetics will appear elsewhere.[4] We note that no evidence for the presence of crystalline structure is observed with highresolution transmission electron microscopy,[8] and that Rutherford backscattering spectroscopy and elastic recoil detection analysis find no evidence for the presence of impurity concentrations to $0.01 \%$ atomic levels.

The evolution in roughness and position of the small angle feature as a function of annealing temperature are related apparently to changes in the films' structure associated with the increase in the ratio of 3-fold to 4-fold coordinated carbon atoms, although this change has been estimated to be a few percent.[1] Other changes contributing to the observed variations are changes at the carbon-substrate interface as the result of the annealing temperatures, as well as the evolution of interfacial layers as a function of temperature. Evidence for the latter is the fact that the films annealed at $500^{\circ} \mathrm{C}$ and $600{ }^{\circ} \mathrm{C}$ are fit equally to a multilayer model.

We have studied structural evolution of a-C films grown using PLD and annealed to $600{ }^{\circ} \mathrm{C}$ using $\mathrm{x}$-ray reflectivity and small angle $\mathrm{x}$-ray scattering. The films' density decrease correlates with the resistivity decrease and stress release observed previously in similarly annealed films. The films' roughness and small angle features reflect changes in the ratio of 3-fold to 4-fold coordinated carbon as well as interfacial evolution in the films. 


\section{References}

1. J. P. Sullivan, T. A. Friedmann, D. R. Tallant, J. Mikkalson, D. J. Rieger, A. G. Baca and L. J. Martínez-Miranda, Appl. Phys. Lett., in press, 1997; J. P. Sullivan, T. A. Friedmann, and A. G. Baca, J. of Elec. Mats., 26, 1026 (1997).

2. Y. Huai et al., Appl. Phys. Lett., 65, 830 (1994).

3. L. J. Martínez-Miranda, J. P. Sullivan, T. A. Friedmann, M. P. Siegal, T. W. Mercer and N. J. DiNardo, Mat. Res. Soc. Symp. Proc., 383, 459 (1995).

4. M. P. Siegal, L. J. Martínez-Miranda et al., in progress (1998).

5. M. P. Siegal , T. A. Friedmann, S. R. Kurtz, D. R. Tallant, R. L. Simpson, F. Dominguez and K. F. McCarty, Mat. Res. Soc. Symp. Proc., 349, 507 (1994).

6. Yushan Shi, unpublished; see also, M. F. Toney and S. Brennan, J. Appl. Phys., 66, 1861 (1989); C. A. Lucas et al., Appl. Phys. Lett., 59, 2100 (1991).

7. T. W. Mercer et al., Mat. Res. Symp. Proc., 358, 863 (1995).

8. J. P. Sullivan and P. P. Newcomer-Provencio, unpublished. 


\section{The Electronic Transport Mechanism in Amorphous Carbon Films}

The electronic transport mechanism in tetrahedrally-coordinated amorphous carbon was investigated using measurements of stress relaxation, thermal evolution of electrical conductivity, and temperature-dependent conductivity measurements. Stress relaxation measurements were used to determine the change in 3-fold coordinated carbon concentration, and the electrical conductivity was correlated to this change. It was found that the conductivity was exponentially proportional to the change in 3-fold concentration, indicating a tunneling or hopping transport mechanism. It was also found that the activation energy for transport decreased with increasing anneal temperature. The decrease in activation energy was responsible for the observed increase in electrical conductivity. A model is described wherein the transport in this material is described by thermally activated conduction along 3-fold linkages or chains with variable range and variable orientation hopping. Thermal annealing leads to chain ripening and a reduction in the activation energy for transport.

Tetrahedrally-coordinated amorphous carbon (a-C, ta-C, a-tC, amorphous diamond, amorphic diamond, etc.) is a medium to wide-gap material (about $1 \mathrm{eV}$ to $2.5 \mathrm{eV}$ ) consisting exclusively of carbon (i.e. no hydrogen) with two types of coordination, 4-fold and 3-fold. The band edges have $\pi$ or $\pi^{*}$ character which originate from the 3 -fold coordinated component (the bond hybridization of an ideal 3-fold coordinated atom being three $\mathrm{sp}^{2}$ bonds and one $\pi$ bond). The magnitude of the band gap is determined primarily by the splitting in energy between the $\pi$ and $\pi^{*}$ states which itself is determined by the degree of linking or clustering of the 3 -fold sites. The configuration (whether chain-like, cluster-like, etc.) of the 3-fold structures embedded in the 4-fold matrix is not known with any certainty. The most energetically favorable configuration is for the 3-fold sites to form compact three-dimensional clusters [1]. However, most recent first principles calculations find that chain-like clustering is the most favored structure with compact (e.g. ring-like) structures being absent [2-4]. This is presumably due to chain-like structures being kinetically easier to form over compact, closed structures.

There are interesting aspects to the electrical properties of ta-C. Despite having a modest band gap, the material does not behave like a conventional semiconductor. The conduction states are localized [5], so the apparent carrier mobilities are quite low. While it is possible to modify the conductivity of these materials by incorporating other elements, e.g. $\mathrm{N}$ [6], the efficiency of these dopants are very low and there is no evidence that it is possible to form pn junctions [7]. The low mobility and inability to create electrical junctions by doping hinders the use of this material in certain device applications, e.g. bi-polar field effect transistors, photocells, 
etc. A more promising application for this material is as an electron emitter material for vacuum field emission devices, e.g. field emission flat panel displays or vacuum microelectronics.

ta- $\mathrm{C}$ also differs from conventional semiconductors in that the current conduction through these materials is spatially inhomogeneous. The measured through-thickness (i.e. perpendicular to the film plane) conductivity varies over a large range (greater than one order of magnitude) with no systematic trends nor correlation with extrinsic defects (particles, etc.) [8]. Interestingly, spatially resolved electron emission from these materials also shows large spatial variation in emission current which could be related to the heterogeneity in conductivity [9].

In this work we discuss the electronic transport mechanism in ta-C films. We use an unconventional approach wherein we measure the change in electrical conductivity in the ta-C film with changes in 3-fold carbon concentration. The change in 3-fold carbon concentration itself is determined by modeling the stress relaxation which occurs in these materials as a result of thermal annealing. The advantage of this approach is that it leads to an unambiguous interpretation of the conduction mechanism, and it permits possible identification of the structural changes which occur in the material following thermal annealing.

The ta-C films used in this study were prepared using pulsed-laser deposition with a $\mathrm{KrF}$ laser and a graphite target. Deposition was performed in vacuum (better than $1 \times 10^{-6}$ Torr) up to a film thickness of 100 to $120 \mathrm{~nm}$ using a laser fluence of $\geq 50 \mathrm{~J} / \mathrm{cm}^{2}$. These deposition conditions led to films with density of $-3.0 \mathrm{~g} / \mathrm{cm}^{3}$, as measured by x-ray reflectivity, corresponding to about $70 \% 4$-fold and $30 \% 3$-fold carbon. For stress relaxation measurements, the films were deposited upon 2" Si wafers which were treated prior to deposition by brief immersion in diluted HF. For electrical measurements, the films were deposited upon a layer of $\mathrm{TiW}$ (200 nm thick) on $\mathrm{SiO}_{2}(1 \mu \mathrm{m})$ on $\mathrm{Si}$.

Thermal annealing was performed ex situ in a rapid thermal annealer in an ambient of flowing Ar. Anneals at successively longer time intervals at the same temperature were performed in order to determine time-dependent changes. The film stress was measured at room temperature following annealing by examining wafer curvature and using Stoney's equation. Contacts ( $\mathrm{Ti}$, followed by $\mathrm{Au}$ ) were photolithographically patterned on to the ta-C film surface and had areas ranging from $2.5 \times 10^{-5} \mathrm{~cm}^{-2}$ to $1.6 \times 10^{-3} \mathrm{~cm}^{-2}$. The DC electrical conductivity of the samples was determined by measuring current-voltage characteristics between a top contact and the TiW layer beneath the ta-C film. A bias range of $-50 \mathrm{mV}$ to $+50 \mathrm{mV}$ was used. Ohmic conduction was observed within this range. Measurement of the activation energies for electrical 
transport were made by performing temperature-dependent conductivity measurements over the range $230 \mathrm{~K}$ to $380 \mathrm{~K}$. No irreversible changes in electrical conductivity of the samples were observed following cycling through this temperature range.

Fig. 4.1(a) shows the measured film stress and normalized conductivity in ta-C films following time-temperature annealing. The film stress drops continuously with annealing from a value of about $7 \mathrm{GPa}$ (compressive) in the as-deposited state to zero after annealing at $600^{\circ} \mathrm{C}$. Most film properties show little or no change following complete stress relaxation at this temperature [10]. The conductivity, Fig. 4.1(b), does show pronounced change, however. Prior to annealing, the conductivity showed considerable variation from contact to contact, but the median value was $\sim 1 \times 10^{-7}(\Omega \mathrm{cm})^{-1}$. Following thermal annealing, the conductivity continued to show large variation from site to site, but after normalization to the initial conductivity, $\sigma_{\text {init }}$, approximate universal behavior was observed for all contacts. The data shown in Fig. 4.1(b) is for contacts that exhibited median behavior.

We have developed a model to explain the stress relaxation which occurs in ta- $\mathrm{C}$ following thermal annealing [8]. The model assumes that thermal annealing causes some 4-fold coordinated carbon atoms to convert to 3 -fold coordination with first order kinetics and a classical activation barrier, $\mathrm{E}_{\mathrm{A}}$ [11]. The stress relaxation arises from the fact that 3 -fold coordinated carbon atoms have, on average, shorter bond lengths than 4-fold coordinated atoms (an $\mathrm{sp}^{2}$ bond is about $8 \%$ shorter than an $\mathrm{sp}^{3}$ bond). The $\pi$ bond of the 3 -fold atom is much longer, however, so that the average volume occupied by a 3 -fold atom is larger than that of a 4fold atom (i.e. conversion leads to a drop in film density [12]). Because of this difference in $\mathrm{sp}^{2}$ and $\mathrm{sp}^{3}$ bond length, strain relief is possible if the newly created 3 -fold atoms are oriented preferentially with the shorter $\mathrm{sp}^{2}$ bonds in the plane of the film. The driving force for this preferential orientation is strain energy: due to the biaxial stress state of the film, there is a reduction in strain energy when 3 -fold sites are created with the preferential orientation while non-optimal orientations, e.g. with the $\pi$ bond in the film plane, cause an increase in strain energy. Finally, because the films are amorphous, there is a distribution of activation energies, $\mathrm{E}_{\mathrm{A}}$, needed to convert 4-fold to 3-fold. In its simplest form, the model time-temperature stress relaxation response is given by

$$
\sigma(t, T)=\sigma_{0}+\frac{\varepsilon_{s p^{3}-s p^{3}} E^{\infty}}{1-v} \int_{0}^{\infty} N\left(E_{A}\right)\left\{1-\exp \left[-v_{0} t \exp \left(-E_{A} / k_{B} T\right)\right]\right\} d E_{A}
$$



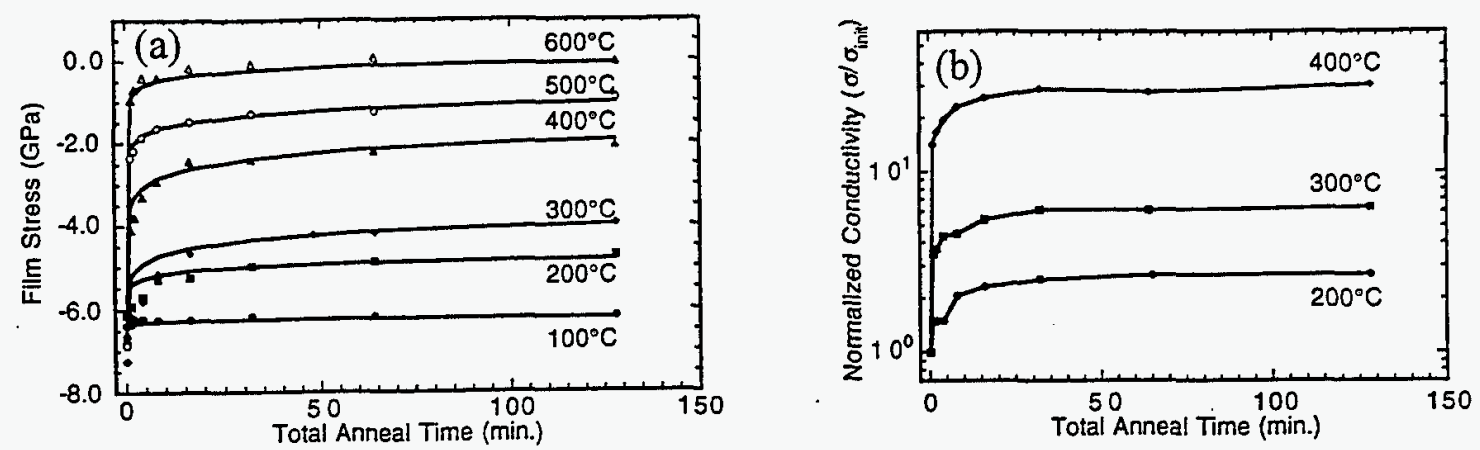

Fig. 4.1: (a) Time and temperature dependence of the stress relaxation in ta-C. Solid lines are fits to the data using the model in the text. (b) Time and anneal temperature dependence of the conductivity change in ta- $C$ (conductivity measured at room temp.).

Solid lines are guides.

where $\sigma(\mathrm{t}, \mathrm{T})$ is the time and temperature-dependent stress, $\sigma_{0}$ is the initial in-plane stress, $\mathrm{E}$ is the elastic modulus of ta-C, $\sim 1000 \mathrm{GPa}$ [13], $v$ is the Poisson ratio, $\sim 0.1, \varepsilon_{s p^{2}-s p^{3}}$ is the strain associated with replacing a 4 -fold atom with a 3 -fold atom $(\sim-0.08), v_{0}$ is the attempt frequency (about $10^{13} \mathrm{sec}^{-1}$ ), and $\mathrm{N}\left(\mathrm{E}_{\mathrm{A}}\right) \mathrm{dE}_{\mathrm{A}}$ is the fraction of 3-fold sites converted from 4-fold sites with an activation energy in the range of $\mathrm{dE}_{\mathrm{A}}$.

Fitting Eq. 1 to the stress relaxation data of Fig. 4.1(a), the distribution of activation energies, $N\left(E_{A}\right)$ was obtained. The activation energies were found to lie in the range of $<1 \mathrm{eV}$ to over $3 \mathrm{eV}$, with a peak near $2 \mathrm{eV}$. Recently, Reznik et al. found an activation energy of 0.7 $\mathrm{eV}$ for conversion of $\mathrm{sp}^{3}$ bonds to $\mathrm{sp}^{2}$ bonds at defect sites in crystalline diamond, which is near the low end of the range observed here [14]. With the measured $N\left(E_{A}\right)$, the change in 3-fold concentration, $\Delta \mathrm{C}_{3 \text {-fold, }}$ can be determined (equal to the integral in Eq. 1). For full stress relaxation, only a small increase in 3fold concentration of about 6.5 at. $\%$ is required, i.e. for an initial 3 -fold concentration of $30 \%$, the concentration following stress relief may increase to as little as $36.5 \%$.

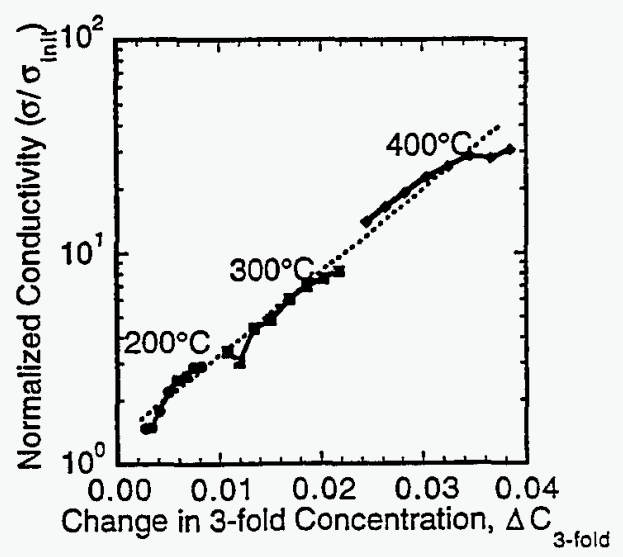

Fig. 2. Electrical conductivity in ta- $C$ as a function of change in 3-fold concentration. The temperatures on the plot indicate the temp. at which the sample was annealed. 

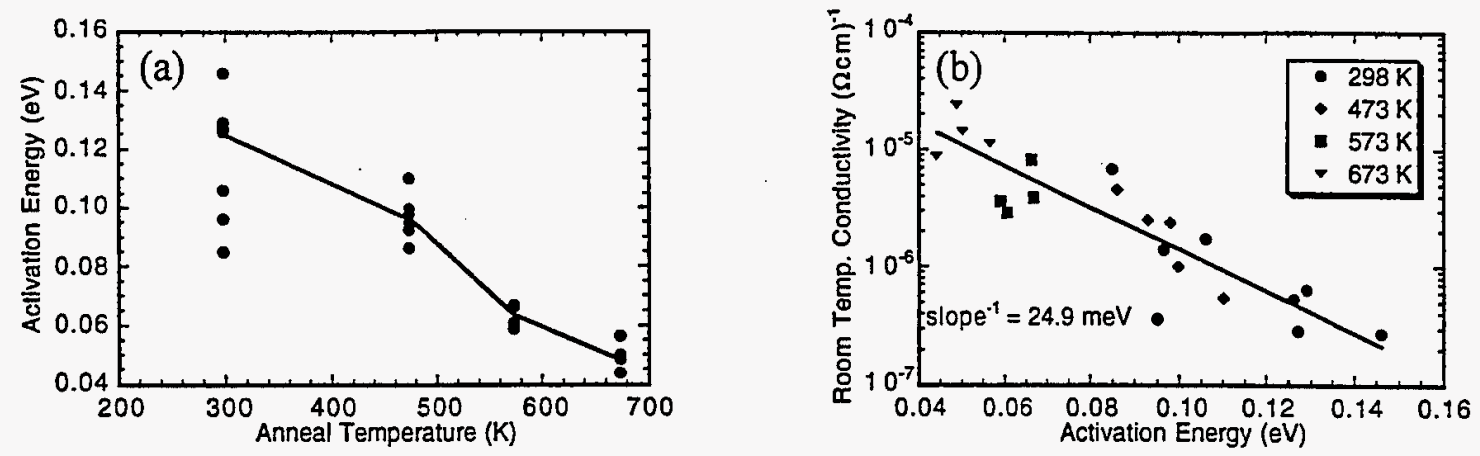

Fig. 4.3: (a) Activation energy for transport for ta-C films annealed at different temperatures. Line is drawn through the median values. (b) Room temperature conductivity as a function of activation energy for annealed and unannealed ta-C films. Solid line is a fit to the data (the slope is reported for a plot of $\ln (\sigma)$ vs. $E_{A}$ ).

The derived change in 3-fold concentration can be combined with the conductivity data in Fig. 4.1 to obtain a correlation between electrical conductivity and 3-fold concentration, Fig. 4.2. An approximately linear relationship was obtained, indicating that

$$
\sigma=\sigma_{\text {init }} \exp \left(\kappa \Delta C_{3-\text { fold }}\right)
$$

where $K \approx 100$. The temperature-dependent conductivity was also measured for several contacts as a function of annealing temperature. Over the temperature range investigated in this study, roughly linear plots were obtained for both $\log$ (conductivity) vs. $1 / \mathrm{T}$ or $\log$ (conductivity) vs $1 / T^{\alpha}$, where $\alpha=1 / 4$, so we can not unambiguously determine the hopping conduction mechanism alone based on temperature-dependent conductivity measurements [15]. Using plots of $\log$ (conductivity) vs. $1 / \mathrm{T}$, we obtain "activation energies" for electronic transport, $\mathrm{E}_{\mathrm{A}}$, and these are shown in Fig. 3(a). Annealing was found to systematically lower the activation energy for transport. Furthermore, the drop in activation energy is sufficient to fully explain the increase in electrical conductivity following annealing, as is shown in Fig. 3(b).

In Fig. 4.3(b), the room temperature conductivity is plotted as a function of the measured activation energy for that particular contact. Data for all contacts and for all annealing temperatures are included. Fitting a line through the data, an inverse slope equal to $24.9 \mathrm{meV}$ was obtained. This is close to the value of $\mathrm{k}_{\mathrm{B}} \mathrm{T}$ at room temperature, which indicates that to good approximation, the conductivity for a particular contact, $i, \sigma_{i}$, is given by 
$\sigma_{i}=\sigma_{0} \exp \left(-E_{A, i} / k_{B} T\right)$, where $\sigma_{0}$ is the pre-exponential factor for electrical transport and is constant, $\approx 9 \times 10^{-5}(\Omega \mathrm{cm})^{-1}$, and $\mathrm{E}_{\mathrm{A}, \mathrm{i}}$ is the activation energy for contact $\mathrm{i}$.

Summarizing these results, (1) the measured electrical conductivity of ta- $\mathrm{C}$ films is exponentially proportional to changes in 3 -fold concentration, $\Delta \mathrm{C}_{3 \text {-fold, }}$, (2) the activation energies for electrical transport are small, but the pre-exponential factors are very small, so the conductivity is low, (3) annealing leads to a systematic reduction in the activation energy for electrical transport, and (4) these changes in apparent activation energy are sufficient to explain the change in conductivity (i.e. the pre-exponential factor does not change). From (1) and (2) it can be concluded that the current conduction mechanism is that of hopping transport [15]. Within the mechanism of hopping conductivity, several possibilities exist for how annealing may

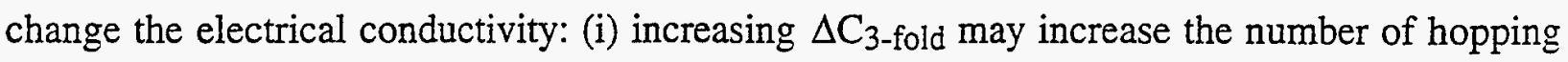
sites, effectively reducing the mean hopping distance, (ii) increasing $\Delta \mathrm{C}_{3 \text {-fold }}$ may lead to an increase in the size of the 3-fold clusters or linkages (e.g. ripening) which would also decrease the hopping distance, or (iii) increasing $\Delta \mathrm{C}_{3 \text {-fold }}$ may lower the activation energy for transport, and this leads to the enhanced conduction. From (3) and (4), above, we can conclude that (i) and (ii) are not the major contributors to the conductivity change with annealing and that the most important effect is (iii).

The reduction in activation energy for electrical transport with thermal annealing may arise from ripening of the 3-fold clusters or linkages. Within the vicinity of a 3-fold cluster or linkage, the local DOS shows an energy gap equal to the energy splitting between the $\pi$ and $\pi^{*}$ states, $E_{\pi}$ $\pi^{*}$. The magnitude of $\mathrm{E}_{\pi-\pi^{*}}$ is a function of the number of carbon atoms in the 3-fold cluster or linkage. For isolated 3-fold chains, Robertson has shown that $\mathrm{E}_{\pi-\pi^{*}}$ varies as $\sim 20 \mathrm{eV} / \mathrm{N}$ where $\mathrm{N}$ is the number of carbon atoms in the chain; a slightly different dependence is obtained if the carbon atoms make compact ring clusters [1]. This dependence of $E_{\pi-\pi^{*}}$ on $N$ is shown in Fig. 4.4. In Fig. 4.4 we also include two data points for recent results of Frauenheim et al. which involve a 6-member and an 8-member 3-fold carbon chain embedded in a 4-fold amorphous carbon matrix, i.e. the calculated structure of ta-C [3]. These two points lie very close to the values calculated by Robertson for isolated chains. 
If we assume that carriers are thermally activated into the $\pi$ or $\pi^{*}$ states of chain-like linkages of 3 -fold atoms, then thermal activation will be easiest (the barrier smallest) for the longest chains (these have the smallest $\mathrm{E}_{\left.\pi-\pi^{*}\right)}$. Hopping conduction therefore constitutes tunneling from long chain to long chain with the activation energy for transport reflecting both the activation energy for transport along the chain (i.e. thermal activation to an extended state of the chain plus the activation energy for propagation

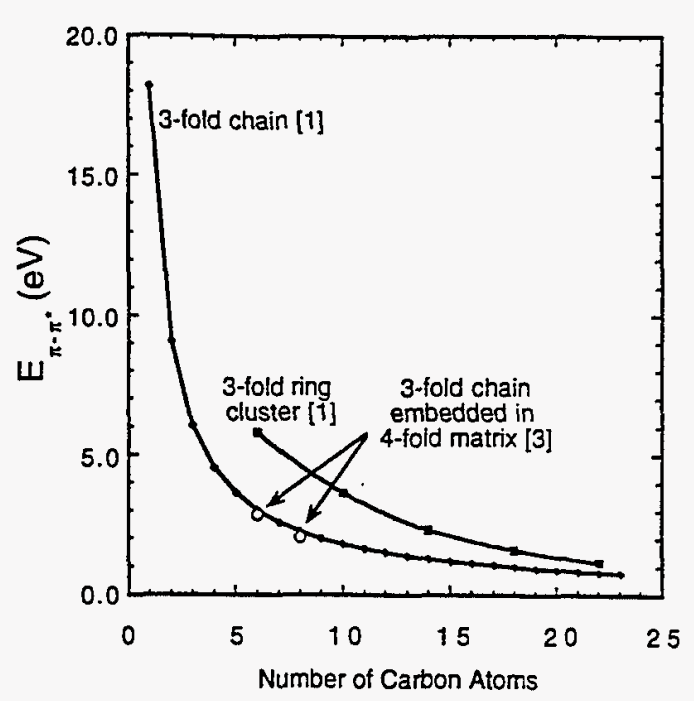
along the chain [16]) as well as the activation energy needed to hop between chains having $\pi$ or $\pi^{*}$ states at different energies. The measured low values for the activation energies suggests that the Fermi level in ta-C likely lies near the $\pi$ states of the longest chains, which is consistent with the reported p-type character of undoped ta$C[6]$.

The view of the hopping conductivity that emerges is as follows: (i) carriers are preferentially activated into the longest 3-fold chains or linkages, (ii) carriers tunnel from long chain to long chain (intervening 3-fold chains have larger $E_{\pi-\pi^{*}}$, and hence their $\pi$ and $\pi^{*}$ states do not overlap with the $\pi$ and $\pi^{*}$ states of the long chains), but it is also necessary that the $\pi$ states of the two long chains have similar orientation (the tunnel probability for orthogonal orientations drops to zero), (iii) thermal annealing causes chain ripening, and (iv) as chains lengthen, $\mathrm{E}_{\pi-\pi^{*}}$ decreases and the activation energy for transport decreases. The hopping conductivity can be thought of as being both variable range and "variable orientation" - this last point referring to the orientational disorder between the $\pi$ conduction states. This disorder is an important part of the conduction process; it has been shown that disorder in the $\pi$ orbital orientation is one of the primary reasons for the strong localization in ta-C [5]. It should also be noted that the conduction process discussed here is considerably different from the nearest neighbor hopping conduction in a-C proposed by Dasgupta $e t$ al. [17].

An implication of this hopping conduction process is that the current conduction process will be highly heterogeneous. Consider a random resistor network model for ta-C. The lowest resistance connections between nodes will be those for which two long chains have similar $\pi$ 
orbital orientations and are in close proximity. These will be relatively rare (optical absorption measurements suggest that only very few large 3 -fold linkages can exist). The optimal current pathways must link up several of these rare low resistance pathways in order to propagate current completely through the film, which is the reason that large spatial variation in conductivity may be observed.

Finally, given the measured relationship between conductivity, $\Delta \mathrm{C}_{3 \text {-fold, and }} \mathrm{E}_{\mathrm{A}}$ (Figs. 4.2 and 4.3), it is possible to determine an estimate for a typical size of a 3-fold chain or linkage responsible for current conduction [18]:

$$
N=\beta^{\prime} / \kappa C_{0} k_{B} T,
$$

where $N$ is the number of carbon atoms in the chain or linkage, $\beta^{\prime}$ is the relationship between activation energy and $\mathrm{N}, \mathrm{K}$ is from Eq. 2 , and $\mathrm{C}_{0}$ is the initial carbon 3-fold concentration (prior to annealing). Using $\beta^{\prime}=0.5 \mathrm{E}_{\pi-\pi^{*}}=10 \mathrm{eV} / \mathrm{N}, \mathrm{K}=100, \mathrm{C}_{0}=0.3$, and $\mathrm{k}_{\mathrm{B}} \mathrm{T}=0.025 \mathrm{eV}$, then we obtain $N \approx 13$ carbon atoms. It can be shown that thermal annealing at $400^{\circ} \mathrm{C}$ need only increase $\mathrm{N}$ from 13 to 14 (i.e. ripening of only one atom), and this would be sufficient to account for the approximate 35 times increase in electrical conductivity. In this estimate, a hypothetical form for $\beta^{\prime}$ (based on $0.5 \mathrm{E}_{\pi-\pi^{*}}$ ) was used; a detailed analysis using a functional form for $\beta^{\prime}$ which is more consistent with the measured activation energies is in preparation.

The nature of the electrical transport mechanism in tetrahedrally-coordinated amorphous carbon films was investigated by studying the dependence of the electrical conductivity on 3-fold carbon concentration and the change in conductivity and transport activation energy following thermal annealing. An exponential dependence of the electrical conductivity on changes in carbon 3-fold content was found, indicating a hopping conduction process. The activation energy for transport was found to decrease with annealing temperature, suggesting ripening of 3fold linkages. The conduction behavior is best described by thermally activated conduction along 3-fold chains or linkages combined with variable range and variable orientation tunneling, primarily between the longest chains or linkages. This conduction process leads to heterogeneity in the current conduction pathways through the sample. 


\section{References}

1. J. Robertson, Adv. Phys. 35, 317 (1986).

2. N. A. Marks, D. R. McKenzie, B. A. Pailthorpe, M. Bernasconi, and M. Parrinello, Phys. Rev. Lett. 76, 768 (1996).

3. Th. Frauenheim, G. Jungnickel, Th. Köhler, P. Stitch, and P. Blaudeck, Proc. 1st Int. Meeting on Amorphous Carbon (World Scientific Publ., Singapore, 1998).

4. P. A. Schultz and E. B. Stechel, to appear in Phys. Rev. B, 1998.

5. J. Robertson, Phil. Mag. B 76, 335 (1997).

6. V. S. Veerasamy, J. Yuan, G. A. J. Amaratunga, W. I. Milne, K. W. R. Gilkes, M. Weiler, and L. M. Brown, Phys. Rev. B 48, 17954 (1993).

7. C. Ronning, U. Griesmeier, M. Gross, H. C. Hofsäss, R. G. Downing, and G. P. Lamaze, Diamond Relat. Mater. 4, 666 (1995).

8. J. P. Sullivan, T. A. Friedmann, and A. G. Baca, J. Electron. Mater. 26, 1021 (1997).

9. N. Missert, T. A. Friedmann, J. P. Sullivan, and R. G. Copeland, Appl. Phys. Lett. 70, 1995 (1997).

10. J. P. Sullivan, T. A. Friedmann, D. R. Tallant, J. Mikkalson, D. Rieger, A. G. Baca, and L. J. Martiñez-Miranda, submitted to Appl. Phys. Lett., 1996.

11. We may also consider $\mathrm{E}_{\mathrm{A}}$ to be an activation energy to re-orient an existing 3-fold site, but since the observed drop in density following annealing is consistent with increasing the 3fold concentration, we will focus on a conversion mechanism.

12. L. J. Martiñez-Miranda, J. P. Sullivan, T. A. Friedmann, M. P. Siegal, and N. J. DiNardo, Mat. Res. Soc. Proc., Vol. 498, 1998.

13. T. A. Friedmann, J. P. Sullivan, J. A. Knapp, D. R. Tallant, D. M. Follstaedt, D. L. Medlin, and P. B. Mirkarimi, Appl. Phys. Lett. 71, 3820 (1997).

14. A. Reznik, V. Richter, and R. Kalish, Phys. Rev. B 56, 7930 (1997).

15. N. F. Mott and E. A. Davis, Electronic Processes in Non-Crvstalline Materials (Clarendon, Oxford, 1971).

16. If phonon modes were soft along the chains, then carriers could self trap within the chain, and there would be an activation energy for polaronic conduction.

17. D. Dasgupta, F. Demichelis, and A. Tafliaferro, Phil. Mag. B 63, 1255 (1991).

18. J. P. Sullivan and T. A. Friedmann, Proc. 1st Int. Meeting on Amorphous Carbon (World Scientific Publ., Singapore, 1998). 


\section{Electron Emission Induced Modifications in Amorphous Carbon}

The cold-cathode electron emission properties of amorphous carbon are promising for flatpanel display and vacuum microelectronics technologies. The onset of electron emission is typically preceded by "conditioning" where the material is stressed by an applied electric field. To simulate conditioning and assess its effect, we combined the spatially-localized field and current of a scanning tunneling microscope tip with high-spatial-resolution characterization. Scanning force microscopy shows that conditioning alters surface morphology and electronic structure. Spatially-resolved electron energy loss spectroscopy indicates that the predominant bonding configuration changes from predominantly fourfold- to threefold-coordination.

Amorphous carbon (a-C) films can be grown with variable amounts of threefold- and fourfold-coordinated $C[1,2]$ by pulsed laser deposition (PLD) [3], cathodic filtered arc deposition [4], or ion beam deposition [5]. a-C films grown at ambient temperatures exhibit high residual stress; thermal processing can be used to relieve the stress without significantly affecting the fourfold-coordinated $C$ content [6]. The hardness and low friction of a-C films can be exploited for use as tribological coatings. a-tC films also exhibit very good cold-cathode electron emission characteristics under relatively low-field conditions [7] making them excellent candidates for applications in novel flat-panel display [8] and vacuum microelectronics [9] technologies.

To initiate emission, as-grown a-C films typically require an electrical "conditioning" process in which a voltage is applied between the film and an anode to set up an electric field. Ramping the voltage at high rates produces micro-discharges that cause significant morphological crater damage [7,9]. It was recently shown [7] that low ramp rates can be used to initiate electron emission without any observable morphological damage using scanning electron microscopy (SEM), however reduced secondary electron emission occurs in the emitting regions. The threshold field for emission current in the initial voltage ramp is typically $>50 \%$ higher than that required after subsequent voltage ramps.

In order to elucidate the nature of potential emission sites further, we have simulated conditioning of a-C films by using the high, spatially-localized electric field and electron flux from a scanning tunneling microscope (STM) tip. We find that ramping the tip-sample bias and current forms stable nanostructures on the order of the tip dimensions $(\sim 100 \mathrm{~nm}$ in extent). These nanostructures reflect both changes in morphology and electronic structure, and their shape is related to the tip geometry. By combining scanning force microscopy (SFM) and 
spatially-resolved electron energy loss spectroscopy (EELS), we conclude that the nanostructures arise after an electron-induced transformation from predominantly fourfold- to threefoldcoordinated $\mathrm{C}$. We infer that these changes are analogous to those that occur during the conditioning process for electron emission [7].

The preparation of the a-C films was similar to that described previously [10]. For this study, $\sim 250 \AA$ thick a-tC films were grown by PLD from solid graphite onto highly-doped Si(111) substrates. $248 \mathrm{~nm}$ ultraviolet radiation from a $\mathrm{KrF}$ excimer laser was incident on the graphite target at an energy density of $27 \mathrm{~J} / \mathrm{cm}^{2}$. Ambient pressure during film growth was $\leq 10^{-7}$ Torr and the substrate temperature was $\leq 50^{\circ} \mathrm{C}$. The conditioning process was simulated and characterized with a Digital Instruments NanoScope III Scanning Probe Microscopy (SPM) system (STM and SFM) operated in air and a Perkin-Elmer Scanning Auger Microprobe (SAM). Complementary measurements were made with a custom-built STM operated in ultrahigh vacuum [11]. The surfaces were conditioned by ramping the STM tip bias at regular points of a grid while scanning over a fresh surface region. The conditioned region was then characterized by re-scanning with the STM or by measuring the structure and other parameters, such as surface potential, with the SFM. Spatially-resolved Auger Electron Spectroscopy (AES) and EELS were also performed over the conditioned regions.

SFM images of as-grown a-C films show that the surfaces are very flat with a root-meansquare roughness of $\leq 1 \mathrm{~nm}$ [12]. Local electronic spectra of the surfaces from current-voltage (I-V) characteristics acquired by STM indicate uniform surface electronic properties. To ensure clean surfaces prior to conditioning, the samples were ultrasonically cleaned in acetone and methanol followed by a $30 \mathrm{~s}$ oxygen plasma etch to remove residual hydrocarbons and graphitic surface species, slightly increasing the roughness [12]. An STM image obtained after electrical conditioning is shown in Figure 5.1. The (incomplete) grid pattern was created during the conditioning STM scan where the feedback was disabled and the sample-tip bias was ramped from $-10 \mathrm{~V}$ to $+10 \mathrm{~V}$ at each grid point over a $10 \times 10 \mu \mathrm{m}^{2}$ region. Not every grid point appears to be modified presumably due to the details of the electric field stress at the location and time of its application. Representative I-V characteristics indicate that when the majority of nanostructures are formed, the tip-to-sample current reaches a value $\geq 100 \mathrm{nA}$. When no change occurs, a significantly reduced forward current is observed. It appears that a sufficiently high electron flux is a key factor to create a change, likely the result of a breakdown in the resistance at the site where the elevated field is applied. In various runs with different tips, the shape of the modified structures changed, indicating a direct correspondence to the shape of the tip. 


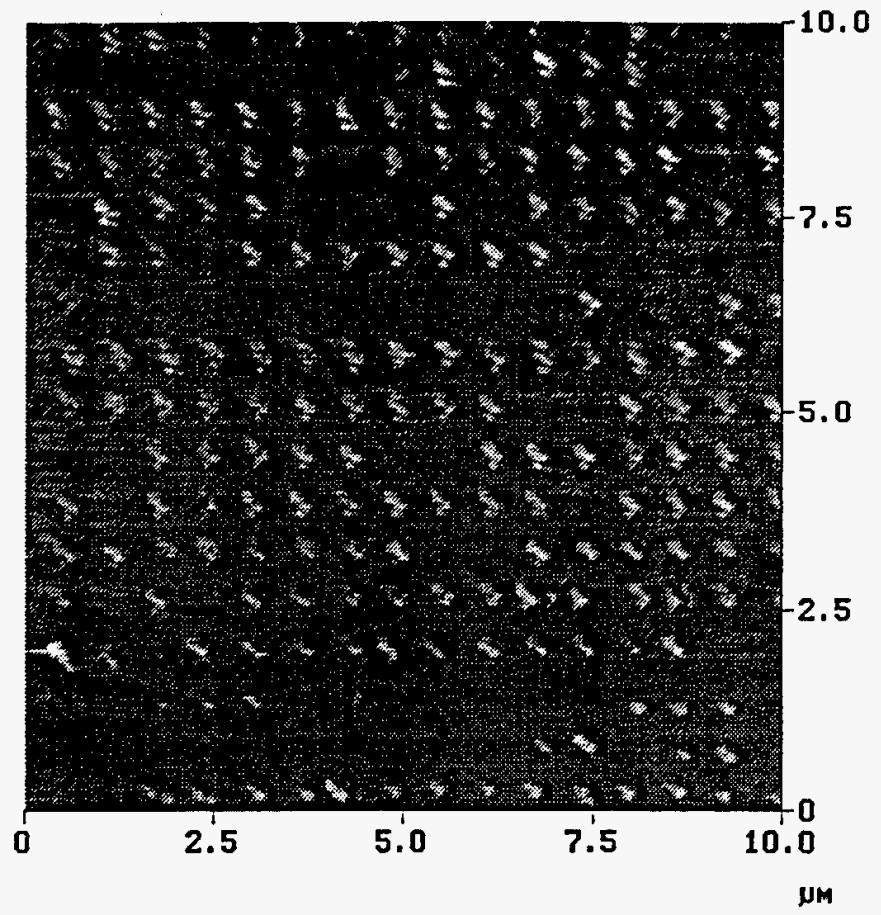

Figure 5.1: STM image after electrical conditioning of the a-C film over a $10 \times 10 \mu \mathrm{m}^{2}$ grid.

Surface chemical composition and near-surface hybridization were probed with the SAM using an a-C film with a higher density grid to increase the sensitivity of the measurement. The conditioned regions could be identified by reduced secondary emission in the SEM mode [7]. Spatially-resolved AES in these regions showed no evidence of impurities $(<0.02$ monolayer sensitivity). Thus, transfer of metal atoms from the tip [13] can be discounted as a possible origin of the observed modification. Conditioning the surface in ultrahigh vacuum indicated similar modification of the film thus excluding the possibility of STM-induced chemical reaction of surface contaminants. In a previous study, transmission EELS was used to distinguish threefold- and fourfold-coordinated $C$ regions with sub-nanometer resolution.[14] Here, spatially-resolved reflection EELS was performed on a modified region to measure the hybridization of the near-surface carbon by analyzing shifts in the energy of the surface plasmon loss.[15] Plasmon excitations centered at $-28 \mathrm{eV}$ are consistent with fourfold-coordinated $\mathrm{C}$ regions and are prevalent in unmodified areas of the sample; plasmon excitations centered at $\sim 26 \mathrm{eV}$ correspond to threefold-coordinated $\mathrm{C}$ regions. Figure 5.2a shows an STM image of a conditioned region; it is seen that a higher success rate for modification was achieved in the upper half of this high-density grid. Figure $5.2 \mathrm{~b}$ presents a $100 \times 100$ point overlay of EELS data (light gray) on the SEM image of the same region. The light gray in the overlay represents areas where the plasmon peak has shifted to lower energies. The plasmon shift correlates with the modified locations in Figure 5.2a indicating that the conditioning is related to an 

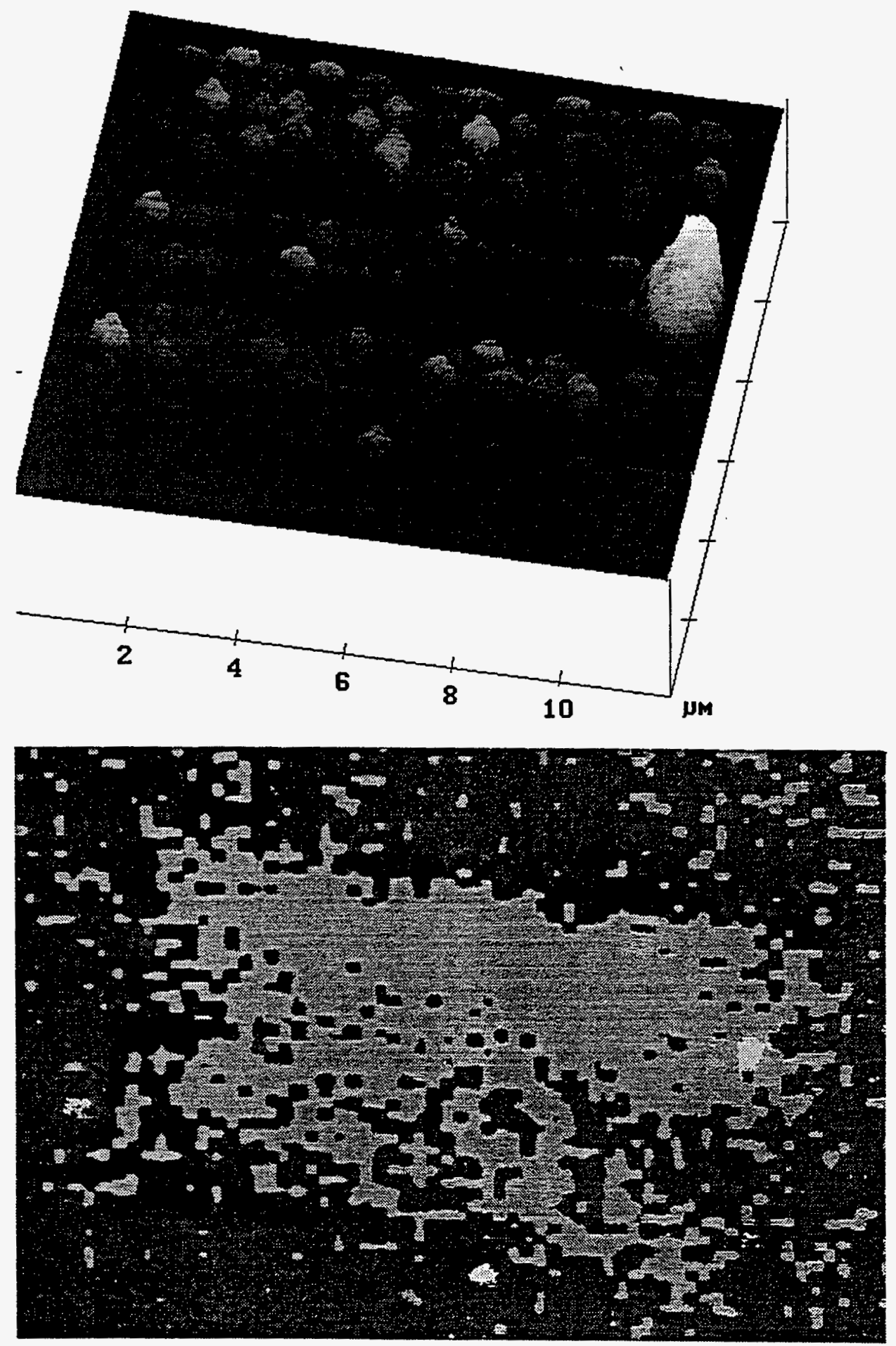

Figure 5.2: (a) STM surface plot $\left(12 \times 12 \mu \mathrm{m}^{2}\right)$ of a locally-modified region of an a-tC film over a $32 \times 32$ point grid; the viewing angle is set to coincide with (b). (b) Overlay of a difference map of plasmon intensities acquired by spatially-resolved EELS over corresponding SEM image. The conditioned region exhibits reduced secondary emission; light gray area highlights the threefold coordination which correlates with the modification in (a). 
increase of threefold-coordinated $C$ species. We note that EELS spectra were stable even after $\sim 4$ hours of SEM imaging suggesting that the films are rather stable under low-flux electron beams.

A distinction can be made between morphological and electronic modification by comparing complementary SPM measurements. Synchronously-acquired intermittent-contact mode SFM and non-contact surface potentiometry images of the grid of Figure 5.1 are presented in Figure 5.3. The sample orientation is different from Figure 5.1 to distinguish possible SFMinduced surface modification. Protrusions of $\sim 20 \mathrm{~nm}$ in the SFM image indicate that modification results in a volumetric expansion of the material which is qualitatively consistent with the formation of lower density threefold-coordinated $C$ from higher density fourfoldcoordinated C. Such small morphological changes would not be seen with SEM. The surface potential was measured at a constant height of $\sim 50 \mathrm{~nm}$ above the surface referenced with respect to the topographic trace. The surface potential of the nanostructures is reduced by $\sim 30 \mathrm{mV}$ with respect to the surrounding region, qualitatively signifying changes in electronic structure. It is important to note that changes in the surface potential occur at some points where no morphological changes occur particularly in the rows marked by arrows suggesting changes in electronic structure alone. It should also be noted that STS indicates that the modified nanostructures exhibit increased conductance for forward biases. In addition, even the nominally unmodified areas between the nanostructures, having been scanned by the STM under typical biases of $<2 \mathrm{~V}$, exhibit subtle but observable changes in surface morphology and surface potential.

Direct electronic excitation or local heating are mechanisms that can cause changes in local bonding in the a-C film. Direct electron-induced interactions have been explored in a study of STM-induced desorption and reconstruction reported for $\mathrm{H}$-terminated $\mathrm{Si}(111)$ surfaces [16]. In that study, high-flux electron bombardment was shown to locally desorb $\mathrm{H}$ leaving behind a metastable $2 \times 1$ surface reconstruction. For the case of a-C films, the issue is rather a local rehybridization of carbon bonds. It is possible that a high electron flux induces $\mathrm{C}-\mathrm{C}$ bond rehybridization in $\mathrm{a}-\mathrm{C}$ materials by electronic excitation. However, since the observed modifications may extend beneath the surface, the possibility that local heating [17] transforms the material to a more stable threefold-coordinated configuration [18] should be considered. Unfortunately, estimates of peak temperatures during conditioning are uncertain because the thermal conductivity of a-C films is generally not known. 


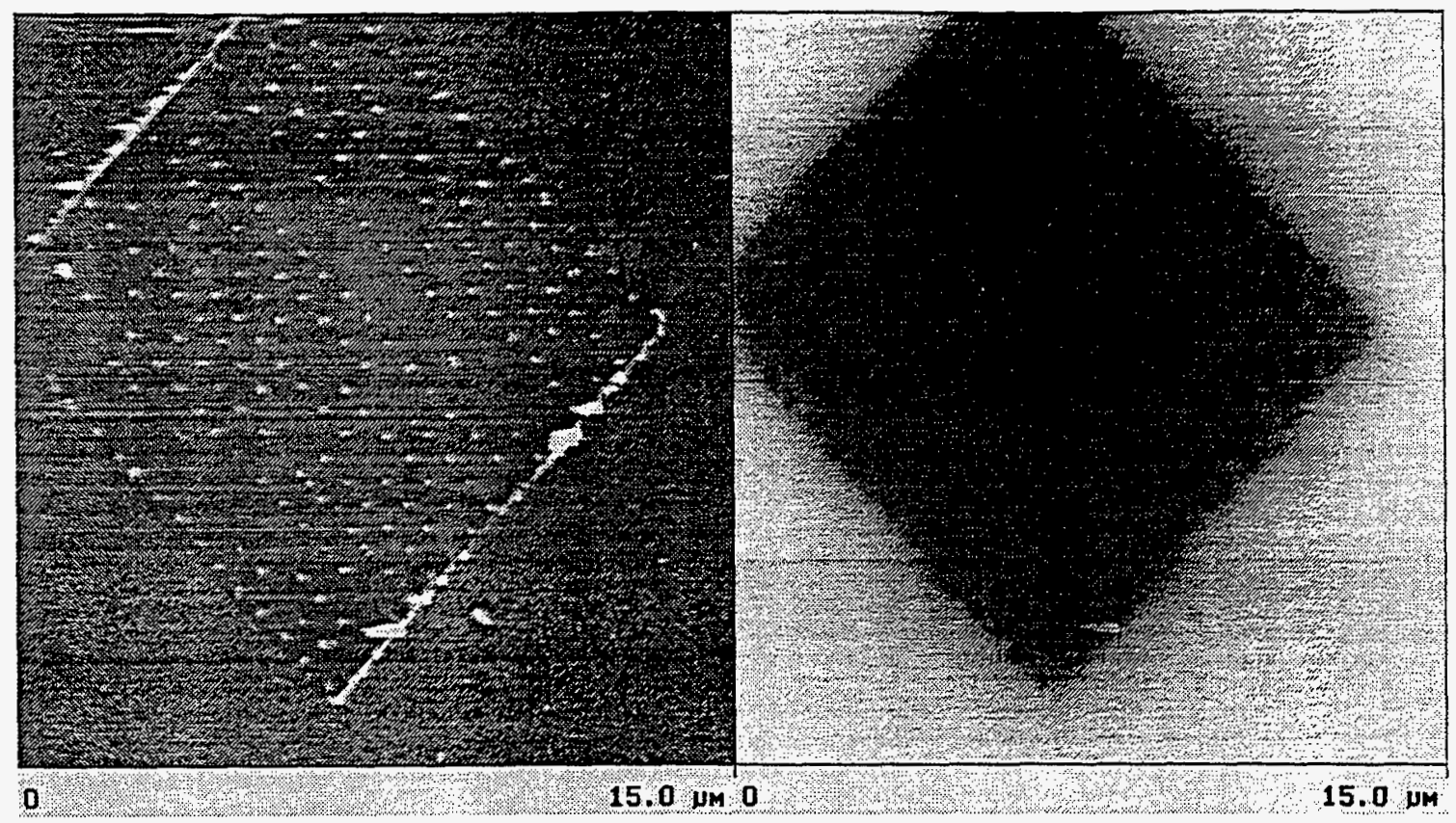

Figure 5.3: Left: Intermittent-contact SFM image of the modified a-tC film shown in Figure 1. Right: SP map acquired synchronously with the SFM image.

This study demonstrates that the process of conditioning a-C films by electric field stress to induce emission can result in structural and electronic changes originating from a change from fourfold- to threefold-coordinated $\mathrm{C}$ bonding configurations. The basis of the thin film modification is local rebonding of $\mathrm{C}$ created by an electron beam flux of sufficient magnitude. 


\section{References}

1. N. A. Marks, D. R. McKenzie, B. A. Pailthorpe, M. Bernasconi and M. Parrinello, Phys. Rev. Lett. 76, 768 (1996).

2. Peter A. Schultz and E. B. Stechel, Phys. Rev. B in press (1998).

3. S. S. Wagal, E. M. Juengerman and C. B. Collins, Appl. Phys. Lett. $\underline{53}, 187$ (1988).

4. P. J. Martin, S. W. Filipczuk, R. P. Netterfield, J. S. Field, D. F. Whitnall and D. R. McKenzie, J. Mat. Sci. Lett. 7, 410 (1988).

5. Srinandan Kasi, Hoen Kang and J. Wayne Rabalais, Phys. Rev. Lett. $\underline{59}, 75$ (1987).

6. J. P. Sullivan, T. A. Friedmann, D. R. Tallant, J. Mikkalson, D. J. Rieger, A. G. Baca and L. J. Martinez-Miranda, Appl. Phys. Lett. (1997).

7. N. Missert, T. A. Friedmann, J. P. Sullivan and R. G. Copeland, Appl. Phys. Lett. $\underline{70}, 1995$ (1997).

8. J. Jaskie, MRS Bull. $\underline{21}, 59$ (1996).

9. A. A. Talin, T. E. Felter, T. A. Friedmann, J. P. Sullivan and M. P. Siegal, J. Vac. Sci. Technol. A 13, 1719 (1996).

10. M. P. Siegal, T. A. Friedmann, S. R. Kurtz, D. R. Tallant, R. L. Simpson, F. Dominguez and K. F. McCarty, in Mater. Res. Soc. Proc. (Materials Research Society, San Francisco, 1994), pp. 507.

11. T. W. Mercer, (Drexel University, 1995),

12. T. W. Mercer, N. J. DiNardo, L. J. Martinez-Miranda, F. Fang, T. A. Friedmann, J. P. Sullivan and M. P. Siegal, in Materials Research Society Symposium on Microcrystalline and Nanocrystalline Semiconductors C. B. Collins, C. C. Tsai, M. Hirose, F. Koch, L. Brus, Eds. (Materials Research Society, Boston, MA, 1994), pp. 863.

13. Gregor S. Hsaio, Reginald M. Penner and Jeff Kingsley, Appl. Phys. Lett. 64, 1350 (1994).

14. David A. Muller, Yujiun Tzou, Rishi Raj and John Silcox, Nature 366, 725 (1993).

15. Fink, $\because$

16. R. S. Becker, G. S. Higashi, Y. J. Chabal and A. J. Becker, Phys. Rev. Lett. 65 , 1917 (1990).

17. U. Staufer, L. Scandella and R. Wiesendanger, Z. Phys. B - Condensed Matter $\underline{77}, 281$ (1989).

18. T. A. Friedmann, K. G. McCarty, J. C. Barbour, M. P. Siegal and Dean C. Dibble, Appl. Phys. Lett. 68, 1643 (1995). 


\section{Summary}

This project set out to study the properties of a-C thin films for possible use in cold-cathode electron emission applications. For electrons to emit from an a-C film surface, they must transport from the film-substrate interface through the film to its surface. Further, the effective work function on the a-C surface must be low enough for electrons to leave the surface under modest applied electric fields. While these films can be grown by a variety of energetic techniques, we used pulsed-laser deposition (PLD) for our work. The laser energy density striking the graphite source target is directly related to the kinetic energy of the ablated carbon species used for film growth. Due to the limited nature of this program (one year), we focussed our study on the following topics:

1) Using Raman spectroscopy, we studied the nature of $\mathrm{C}-\mathrm{C}$ bonding in a-C films as a function of PLD growth energetics.

2) Using $\mathrm{x}$-ray reflectivity, we studied structural changes in a-C films resulting from postannealing experiments designed to simulate typical device processing conditions.

3) Performed electrical resistivity measurements to understand transport in a-C. Taken together with measurements of stress-relief which results from post-annealing, a model for conductivity is established.

4) Using scanning atom probes, we directly studied localized emission from a-C films. More importantly, we were able to simulate a process known as "preconditioning," necessary for emission from a-C films, and for the first time, established the structural changes in bonding that occur. 
Distribution:

$\begin{array}{lll}1 & \text { MS-0188 } & \text { C. E. Meyers, 4523 } \\ 1 & \text { MS-1411 } & \text { D. R. Tallant, 1812 } \\ 1 & \text { MS-1411 } & \text { R. L. Simpson, 1812 } \\ 1 & \text { MS-1421 } & \text { E. B. Stechel, 1153 } \\ 2 & \text { MS-1421 } & \text { M. P. Siegal, 1153 } \\ 1 & \text { MS-1421 } & \text { J. P. Sullivan, 1153 } \\ 1 & \text { MS-1427 } & \text { S. T. Picraux, 1100 } \\ 1 & \text { MS-9018 } & \text { Central Technical Files, 8940-2 } \\ 2 & \text { MS-0899 } & \text { Technical Library, 4916 } \\ 2 & \text { MS-0619 } & \text { Review \& Approval Desk, } 12690 \text { (for DOE/OSTI) }\end{array}$




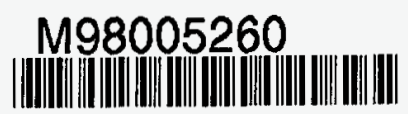

Report Number (14)

SAND $-98-10 / 3$

Publ. Date (11) $\quad 199805$

Sponsor Code (18) DOE/ER

UC Category (19) UC-404,DOE/ER

\section{9}

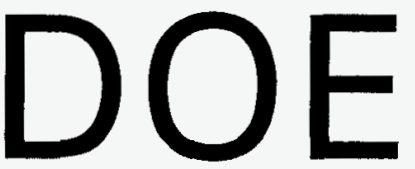

